\title{
Lattice Point Asymptotics and Volume Growth on Teichmüller Space.
}

\author{
Jayadev Athreya*, Alexander Bufetov ${ }^{\dagger}$ Alex Eskin ${ }^{\ddagger}$ and Maryam Mirzakhani ${ }^{\S}$
}

September 20, 2011

\begin{abstract}
We apply some of the ideas of the Ph.D. Thesis of G. A. Margulis Mar70 to Teichmüller space. Let $X$ be a point in Teichmüller space, and let $B_{R}(X)$ be the ball of radius $R$ centered at $X$ (with distances measured in the Teichmüller metric). We obtain asymptotic formulas as $R$ tends to infinity for the volume of $B_{R}(X)$, and also for the cardinality of the intersection of $B_{R}(X)$ with an orbit of the mapping class group.
\end{abstract}

\section{Introduction.}

Much of the study of the geometry and dynamics on Teichmüller and moduli spaces is inspired by analogies with negatively curved spaces. Two classical problems in the negative curvature setting are the questions of lattice point counting and volume growth entropy. Let $M$ be a compact negatively curved Riemannian manifold, and $\widetilde{M}$ its universal cover. $\Pi=\pi_{1}(M)$ acts on $\widetilde{M}$ by isometries. Let $m_{B M}$ denote the Bowen-Margulis measure on $\widetilde{M}$. Given $X, Y \in \widetilde{M}, R>0$, let $B_{R}(X) \subset \widetilde{M}$ be the ball of radius $R$ centered at X. Let $p: \widetilde{M} \rightarrow M$ be the natural projection.

In his Ph.D. thesis G. A. Margulis [Mar70] showed:

Theorem 1.1 There is a function $c: M \times M \rightarrow \mathbb{R}^{+}$so that for every $X, Y \in \widetilde{M}$,

$$
\begin{gathered}
\left|\Pi \cdot Y \cap B_{R}(X)\right| \sim c(p(X), p(Y)) e^{h R} \\
m_{B M}\left(B_{R}(X)\right) \sim\left(\int_{M} c(p(X), z) d m_{B M}(z)\right) e^{h R}
\end{gathered}
$$

where $h>0$ is the topological entropy of the geodesic flow. Here and below, the notation $A \sim B$ means that $A / B \rightarrow 1$ as $R \rightarrow \infty$.

*partially supported by NSF grants DMS 0603636, DMS 0244542 and DMS 1069153

${ }^{\dagger}$ A.I.B. is an Alfred P. Sloan Research Fellow. He is supported in part by Grant MK-4893.2010.1 of the President of the Russian Federation, by the Programme on Mathematical Control Theory of the Presidium of the Russian Academy of Sciences, by the Programme 2.1.1/5328 of the Russian Ministry of Education and Research, by the Edgar Odell Lovett Fund at Rice University, by the NSF under grant DMS 0604386, and by the RFBR-CNRS grant 10-01-93115.

${ }^{\ddagger}$ partially supported by NSF grants DMS 0244542, DMS 0604251 and DMS 0905912

$\S$ partially supported by the Clay foundation and by NSF grant DMS 0804136. 
In this paper, we apply some of the ideas of Margulis from Mar70 to the problems of counting lattice points and understanding volume growth entropy in Teichmüller space. Our main results (Theorems 1.2 and 1.3) are analogues of Theorem 1.1 for Teichmüller space, and the fact that the Bowen-Margulis measure coincides with the Lebesgue measure (see $\$ 2.2$ ). In the rest of this introduction we give the required background and definitions of Teichmüller space and quadratic differentials, and state our main results.

\subsection{Teichmüller space and quadratic differentials.}

We briefly recall some defintions. For a more detailed introduction, see, e.g., FaMa. Let $g \geq 2$, and let $\Sigma_{g}$ be a compact topological surface of genus $g$. Let $\mathcal{M}_{g}$ and $\mathcal{T}_{g}$ denote the moduli space and the Teichmüller space of compact Riemann surfaces of genus $g$.

That is, $\mathcal{T}_{g}$ is the space of equivalence classes of pairs $(X, f)$ where $X$ is a compact genus $g$ Riemann surface and $f: \Sigma_{g} \rightarrow X$ is a diffeomorphism (known as a marking). The equivalence relation is given by $(X, f) \approx(Y, h)$ if there is a biholomorphism $\phi: X \rightarrow Y$ so that $h^{-1} \circ \phi \circ f$ is isotopic to the identity.

Let $\Gamma$ be the mapping class group of $\Sigma_{g}$, given by isotopy classes of orientation preserving diffeomorphisms of $\Sigma_{g}$. That is, $\Gamma=\operatorname{Diff}^{+}\left(\Sigma_{g}\right) / \operatorname{Diff}_{0}^{+}\left(\Sigma_{g}\right)$. $\Gamma$ acts on $\mathcal{T}_{g}$ by changing the marking, and we have $\mathcal{M}_{g}=\mathcal{T}_{g} / \Gamma$.

$\mathcal{T}_{g}$ carries a natural Finsler metric invariant under $\Gamma$, known as the Teichmüller metric. It is given by measuring the quasi-conformal dilatation between surfaces, see, e.g. FaMa. The cotangent space of $\mathcal{T}_{g}$ at a point $X$ can be identified with the vector space $Q(X)$ of holomorphic quadratic differentials on $X$. Recall that given $X \in \mathcal{T}_{g}$, a quadratic differential $q \in Q(X)$ is a tensor locally given by $\phi(z) d z^{2}$ where $\phi$ is holomorphic with respect to the complex structure given by $X$. Then the space $\mathcal{Q T}_{g}=\left\{(q, X) \mid X \in \mathcal{T}_{g}, q \in Q(X)\right\}$ is the cotangent space of $\mathcal{T}_{g}$. In this setting, the Teichmüller metric corresponds to the norm

$$
\|q\|_{\mathcal{T}}=\int_{X}|\phi(z)||d z|^{2}
$$

on $\mathcal{Q} \mathcal{T}_{g}$.

Let $\mathcal{Q}^{1} \mathcal{M}_{g}$ (resp. $\mathcal{Q}^{1} \mathcal{T}_{g}$ ) denote the bundle of unit-norm holomorphic quadratic differentials on $\mathcal{M}_{g}$ (resp. $\mathcal{T}_{g}$ ). We have $\mathcal{Q}^{1} \mathcal{M}_{g}=\mathcal{Q}^{1} \mathcal{T}_{g} / \Gamma$.

The space $\mathcal{Q}^{1} \mathcal{T}_{g}$ carries a natural smooth measure $\mu$, preserved by the action of $\Gamma$ and such that

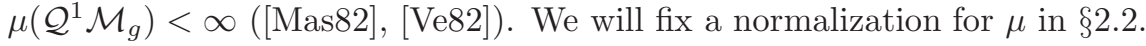

We have a natural projection

$$
\pi: \mathcal{Q}^{1} \mathcal{T}_{g} \rightarrow \mathcal{T}_{g}
$$

and we set $\mathbf{m}=\pi_{*} \mu$.

\subsection{Statement of results.}

Let $X, Y \in \mathcal{T}_{g}$ be arbitrary points, and let $B_{R}(X)$ be the ball of radius $R$ in the Teichmüller space in the Teichmüller metric, centered at the point $X$.

Our main results are the following two theorems: 
Theorem 1.2 (Lattice Point Asymptotics) As $R \rightarrow \infty$,

$$
\left|\Gamma \cdot Y \cap B_{R}(X)\right| \sim \frac{1}{h \mathbf{m}\left(\mathcal{M}_{g}\right)} \Lambda(X) \Lambda(Y) e^{h R},
$$

where $h=6 g-6$ is the entropy of the Teichmüller geodesic flow with respect to Lebesgue measure (see [Ve86]), and $\Lambda$ is a bounded function called the Hubbard-Masur function, which we define in 丹2.3.

Theorem 1.3 (Volume Asymptotics) As $R \rightarrow \infty$,

$$
\mathbf{m}\left(B_{R}(X)\right) \sim \frac{1}{h \mathbf{m}\left(\mathcal{M}_{g}\right)} e^{h R} \Lambda(X) \cdot \int_{\mathcal{M}_{g}} \Lambda(Y) d \mathbf{m}(Y) .
$$

We also prove versions of the above theorems in "sectors", see Theorem 2.9. Theorem 2.10 and Theorem 5.2 below.

Acknowledgments. The authors are grateful to Giovanni Forni, Vadim Kaimanovich, Yair Minsky and Kasra Rafi for useful discussions, and especially to Howard Masur for his help with all parts of the paper, in particular the appendix. We are also grateful to the Institute for Advanced Study, IHES and MSRI for their support.

\subsection{Organization of the paper.}

This paper is organized as follows: In \$2 we describe the main ingredients in the proofs of our main theorems. We construct foliations and measures satisfying the Margulis property of uniform expansion in $\$ 2.2$ We define the Hubbard-Masur function $\Lambda$ and describe its basic properties and relation to counting multicurves in $\$ 2.3$. In $\$ 2.4$ we state a crucial lemma, Proposition 2.5 , on measure in polar coordinates. We postpone the proof to $\$ 3.2$ In $\$ 2.5$ we show how to use mixing to obtain equidistribution results, and apply them to the counting problem in $\$ 2.6$. In $\$ 3$ we recall the definition of the Hodge norm for abelian differentials (\$3.1), and extend and modify it to quadratic differentials (\$3.3). We use this to define a distance along leaves of the stable and unstable foliations for the Teichmüller geodesic flow, and compare it to other distances on $\mathcal{T}_{g}$ in $\$ 3.5$. We prove a non-expansion result for this distance, Theorem 3.15, in $\$ 3.6$ In 3 , we use the results of $\$ 3$ to prove the key estimates Theorem 2.6 and Theorem 2.7 from \$2.5. Finally, in \$5. we prove our volume asymptotics result, Theorem 1.3 and relate it to counting multicurves.

\section{Outline of Proof.}

\subsection{Notation and background.}

Teichmüller geodesic flow. We recall that when $g>1$, the Teichmüller metric is not Riemannian. However, geodesics in this metric are well understood. A quadratic differential $q \in \mathcal{Q} \mathcal{T}_{g}$ with zeros at $x_{1}, \ldots, x_{k}$ is determined by an atlas of charts $\left\{\phi_{i}\right\}$ mapping open subsets of $\Sigma_{g}-\left\{x_{1}, \ldots, x_{k}\right\}$ to $\mathbb{R}^{2}$ such that the change of coordinates are of the form $v \rightarrow \pm v+c$. Therefore the group $\mathrm{SL}_{2}(\mathbb{R})$ acts naturally on $\mathcal{Q M}_{g}$ by acting on the corresponding atlas; given $A \in \mathrm{SL}_{2}(\mathbb{R}), A \cdot q \in \mathcal{Q M}_{g}$ is determined by the new atlas $\left\{A \phi_{i}\right\}$. 
Let $g_{t}=\left[\begin{array}{cc}e^{t} & 0 \\ 0 & e^{-t}\end{array}\right]$. The action of the diagonal subgroup $\left\{g_{t} \mid t \in \mathbb{R}\right\}$ is the Teichmüller geodesic flow for the Teichmüller metric (see [FaMa]).

Warning. In our normalization for the Teichmüller metric, the Teichmüller distance between $\pi\left(g_{t} q\right)$ and $\pi(q)$ is $t$. This normalization (and thus our value for the entropy $h$ ) differs by a factor of 2 from that of e.g. Ve86. Our normalization is chosen in such a way that the top Lyapunov exponent of the Kontsevich-Zorich cocycle is equal to one. In this case the top Lyapunov exponent of the flow is equal to two. For a detailed discussion of the connection between the Lyapunov exponents of the Teichmüller geodesic flow and the Kontsevich-Zorich cocycle, see, e.g., Fo02, where the same speed normalization is used for the Teichmüller geodesic flow as in our paper.

We have Ve82, Mas82:

Theorem 2.1 (Veech, Masur) The space $\mathcal{Q}^{1} \mathcal{M}_{g}$ carries a unique up to normalization measure $\mu$ in the Lebesgue measure class such that:

- $\mu\left(\mathcal{Q}^{1} \mathcal{M}_{g}\right)<\infty$.

- the action of $S L_{2}(\mathbb{R})$ is volume preserving and ergodic;

- the Teichmüller geodesic flow is mixing.

Extremal lengths. Let $X$ be a Riemann surface. Then the extremal length of a simple closed curve $\gamma$ on $X$ is defined by

$$
\operatorname{Ext}_{\gamma}(X)=\sup _{\rho} \frac{\ell_{\gamma}(\rho)^{2}}{\operatorname{Area}(X, \rho)}
$$

where the supremum is taken over all metrics $\rho$ conformally equivalent to $X$, and $\ell_{\gamma}(\rho)$ denotes the length of $\gamma$ in the metric $\rho$. The extremal length can be extended continuously from the space of simple closed curves to the space of $\mathcal{M F}$ of measured foliations, in such a way that $\operatorname{Ext}_{t \beta}(X)=$ $t^{2} \operatorname{Ext}_{\beta}(X)$ Ker80. On the other hand, by the uniformization theorem, each point $X \in \mathcal{T}_{g}$ has a complete hyperbolic metric $\rho_{0}$ of constant curvature -1 in its conformal class. In general, for any simple closed curve $\alpha$,

$$
\frac{\operatorname{Ext}_{\alpha}(X)}{\ell_{\alpha}(X)} \leq \frac{1}{2} e^{\ell_{\alpha}(X) / 2}
$$

where $\ell_{\alpha}(X)$ is the length of the geodesic representative of $\alpha$ on $X$ with respect to the hyperbolic metric $\rho_{0}$ Mas. Also, given $X$ there exists a constant $C_{X}$ such that

$$
\frac{1}{C_{X}} \ell_{\alpha}(X) \leq \sqrt{\operatorname{Ext}_{\alpha}(X)} \leq C_{X} \ell_{\alpha}(X) .
$$

The following result [Ker80 relates the ratios of extremal lengths to the Teichmüller distance:

Theorem 2.1 (Kerckhoff) Given $X, Y \in \mathcal{T}_{g}$, the Teichmüller distance between $X$ and $Y$ is given by

$$
d_{\mathcal{T}}(X, Y)=\sup _{\beta \in \mathcal{C}} \log \left(\frac{\sqrt{\operatorname{Ext}_{\beta}(X)}}{\sqrt{\operatorname{Ext}_{\beta}(Y)}}\right)
$$

where $\mathcal{C}$ is the set of simple closed curves on $\Sigma_{g}$. 


\subsection{The Margulis property.}

Let $g_{t}$ be the Teichmüller geodesic flow on $\mathcal{Q}^{1} \mathcal{T}_{g}$. Note that $g_{t}$ commutes with the action of $\Gamma$ and preserves the measure $\mu$. We will be using the mixing property of the dynamical system $\left(g_{t}, \mathcal{Q}^{1} \mathcal{M}_{g}, \mu\right)$ in $\$ 2.5$

Recall that a quadratic differential $q$ is uniquely determined by its imaginary and real measured foliations $\eta^{-}(q)$ given by $\operatorname{Im}\left(q^{1 / 2}\right)$ and $\eta^{+}(q)$ given by $\operatorname{Re}\left(q^{1 / 2}\right)$. In this notation, we have $g_{t} q=$ $g_{t}\left(\eta^{+}(q), \eta^{-}(q)\right)=\left(e^{t} \eta^{+}(q), e^{-t} \eta^{-}(q)\right)$. See e.g. [FLP] for more details on measured foliations.

The flow $g_{t}$ preserves the following foliations:

1. $\mathcal{F}^{s s}$, whose leaves are sets of the form $\left\{q: \eta^{+}(q)=\right.$ const $\}$;

2. $\mathcal{F}^{u u}$, whose leaves are sets of the form $\left\{q: \eta^{-}(q)=\right.$ const $\}$.

In other words, for $q_{0} \in \mathcal{Q}^{1} \mathcal{T}_{g}$, a leaf of $\mathcal{F}^{s s}$ is given by

$$
\alpha^{s s}\left(q_{0}\right)=\left\{q \in \mathcal{Q}^{1} \mathcal{T}_{g}: \eta^{+}(q)=\eta^{+}\left(q_{0}\right)\right\},
$$

and a leaf of $\mathcal{F}^{u u}$ is given by

$$
\alpha^{u u}\left(q_{0}\right)=\left\{q \in \mathcal{Q}^{1} \mathcal{T}_{g}: \eta^{-}(q)=\eta^{-}\left(q_{0}\right)\right\} .
$$

Note that the foliations $\mathcal{F}^{s s}, \mathcal{F}^{u u}$ are invariant under both $g_{t}$ and $\Gamma$; in particular, they descend to the moduli space $\mathcal{Q}^{1} \mathcal{M}_{g}$.

We also consider the foliations $\mathcal{F}^{u}$ whose leaves are defined by

$$
\alpha^{u}(q)=\bigcup_{t \in \mathbb{R}} g_{t} \alpha^{u u}(q)
$$

and $\mathcal{F}^{s}$ whose leaves are defined by

$$
\alpha^{s}(q)=\bigcup_{t \in \mathbb{R}} g_{t} \alpha^{s s}(q) .
$$

Denote by $p: \mathcal{M F} \rightarrow \mathcal{P} \mathcal{M F}$ the natural projection from the space of measured foliations onto the space of projective measured foliations. Now we may write

$$
\begin{aligned}
& \alpha^{s}\left(q_{0}\right)=\left\{q \in \mathcal{Q}^{1} \mathcal{T}_{g}: \quad p\left(\eta^{+}(q)\right)=p\left(\eta^{+}\left(q_{0}\right)\right)\right\} ; \\
& \alpha^{u}\left(q_{0}\right)=\left\{q \in \mathcal{Q}^{1} \mathcal{T}_{g}: \quad p\left(\eta^{-}(q)\right)=p\left(\eta^{-}\left(q_{0}\right)\right)\right\} .
\end{aligned}
$$

The foliations $\mathcal{F}^{u}$ and $\mathcal{F}^{s s}$ form a complementary pair in the sense of Margulis Mar70 (so do $\mathcal{F}^{s}$ and $\mathcal{F}^{u u}$, but the first pair will be more convenient for us). Note that the foliations $\mathcal{F}^{s s}, \mathcal{F}^{s}, \mathcal{F}^{u}, \mathcal{F}^{u u}$ are, respectively, the strongly stable, stable, unstable, and strongly unstable foliations for the Teichmüller flow in the sense of Veech [Ve86] and Forni [Fo02. (See Theorem 3.15 below for further results in this direction).

Conditional measures. The main observation, lying at the center of our construction, is the following. Each leaf $\alpha^{u u}$ of the foliation $\mathcal{F}^{u u}$, as well as each leaf $\alpha^{s s}$ of the foliation $\mathcal{F}^{s s}$ carries a globally defined normalized conditional measure $\mu_{\alpha^{s s}}$, invariant under the action the mapping class group, and having, moreover, the following property: 


$$
\begin{aligned}
& \text { 1. }\left(g_{t}\right)_{*} \mu_{\alpha^{u u}}=\exp (-h t) \mu_{g_{t} \alpha^{u u}} ; \\
& \text { 2. }\left(g_{t}\right)_{*} \mu_{\alpha^{s s}}=\exp (h t) \mu_{g_{t} \alpha^{s s}} .
\end{aligned}
$$

where $h=6 g-6$ is the entropy of the flow $g_{t}$ on $\mathcal{Q}^{1} \mathcal{M}_{g}$ with respect to the smooth measure $\mu$.

The measures $\mu_{\alpha^{u u}}$ and $\mu_{\alpha^{s s}}$ may be constructed as follows. Let $\nu$ denote the Thurston measure on $\mathcal{M F}$ [FLP]. Note that each leaf $\alpha^{s}$ of $\mathcal{F}^{s}$ is homeomorphic to an open subset of $\mathcal{M F}$ via the map $\eta^{-}$. We can thus define the conditional measure on this leaf to be the pullback of $\nu$, denote this measure by $\mu_{\alpha^{s}}$. Similarly one can define the conditional measures $\mu_{\alpha^{u}}$ on leaves $\alpha^{u}$ of $\mathcal{F}^{u}$.

To define the measures on leaves of $\mathcal{F}^{s s}$ (and $\mathcal{F}^{u u}$ ), we restrict the conditional measure from a leaf of $\mathcal{F}^{s}$ to a leaf of $\mathcal{F}^{s s}$ (and similarly from a leaf of $\mathcal{F}^{u}$ to a leaf of $\mathcal{F}^{u u}$ ). This can be done explicitly in the following way: for a subset $E \subset \mathcal{M F}$, let $C o n e(E)$ denote the cone based at the origin and ending at $E$ (i.e. the union of all the line segments connecting the origin and points of $E)$. We write $\bar{\nu}(E)$ to denote $\nu(C o n e(E))$. Now for a set $F \subset \alpha^{s s}$, we define $\mu_{\alpha^{s s}}(F)=\bar{\nu}\left(\eta^{-}(F)\right)$. Similarly, for $F \subset \alpha^{u u}, \mu_{\alpha^{u u}}(F)$ is defined to be $\bar{\nu}\left(\eta^{+}(F)\right)$.

In particular, if $\alpha_{1}$ and $\alpha_{2}$ are two leaves of the foliation $\mathcal{F}^{u}$ and $U_{1} \subset \alpha_{1}$ and $U_{2} \subset \alpha_{2}$ are chosen in such a way that $\eta^{+}\left(U_{1}\right)=\eta^{+}\left(U_{2}\right)$, then we have $\mu_{\alpha_{1}}\left(U_{1}\right)=\mu_{\alpha_{2}}\left(U_{2}\right)$. The equality $\eta^{+}\left(U_{1}\right)=\eta^{+}\left(U_{2}\right)$ is equivalent to the statement that $U_{1}$ may be taken to $U_{2}$ by holonomy along the leaves of the strongly stable foliation $\mathcal{F}^{s s}$; the equality $\mu_{\alpha_{1}}\left(U_{1}\right)=\mu_{\alpha_{2}}\left(U_{2}\right)$ thus means that the smooth measure $\mu$ has the property of holonomy invariance with respect to the pair of foliations $\left(\mathcal{F}^{u}, \mathcal{F}^{s s}\right)$.

This construction allows us to apply the arguments of G.A. Margulis and to compute the asymptotics for the volume of a ball of growing radius in Teichmüller space as well as the asymptotics of the number of elements in the intersection of a ball with the orbit of the mapping class group. The approach is similar, as noted above, to that of EMc93.

Normalization of $\mu$. For convenience, we normalize the measure $\mu$ so that locally $d \mu=d \mu_{\alpha^{u}} d \mu_{\alpha^{s s}}=$ $d \mu_{\alpha^{s}} d \mu_{\alpha^{u u}}$.

\subsection{The Hubbard-Masur function.}

The Hubbard-Masur Theorem. The Hubbard-Masur Theorem HuMas79 states that given any point $X \in \mathcal{T}_{g}$ and any measured foliation $\beta \in \mathcal{M F}$, there exists a unique holomorphic quadratic differential $q$ on $X$ such that $\eta^{+}(q)=\beta$. We also have the identity $\operatorname{Area}(q)=\operatorname{Ext}_{\beta}(X)$.

The measure $s_{X}$ and the multiple zero locus. For $X \in \mathcal{T}_{g}$, we consider the unit (co)-tangent sphere $S(X)=\left\{q \in \mathcal{Q}^{1} \mathcal{T}_{g}: \pi(q)=X\right\}$ at the point $X$. The conditional measure of $\mu$ on the sphere $S(X)$ will be denoted by $s_{X}$. It is by definition normalized so that $s_{X}(S(X))=1$.

Let $\mathcal{P}(1, \ldots, 1) \subset \mathcal{Q}^{1} \mathcal{T}_{g}$ denote the subset where the all zeroes of the quadratic differential are distinct. $\mathcal{P}(1, \ldots, 1)$ is called the principal stratum. Its complement in $\mathcal{Q}^{1} \mathcal{T}_{g}$ is the multiple zero locus. It is easy to see that the measures $s_{X}$ are defined for all $X$ and that this family of measures is smooth away from the multiple zero locus. We will also need the following:

Theorem 2.2 For any $X \in \mathcal{T}_{g}$, the measure $s_{X}$ gives zero weight to the multiple zero locus.

Proof. See appendix A.

The Hubbard-Masur function. Let $q \in \mathcal{Q}^{1} \mathcal{T}_{g}$ and let $\alpha^{u}(q)$ be the leaf of the foliation $\mathcal{F}^{u}$ containing $q$. By the Hubbard-Masur Theorem, the projection $\pi$ induces a continuous bijection 
between $\alpha^{u}(q)$ and $\mathcal{T}_{g}$ which is smooth away from the multiple zero locus. The mapping $\pi$ thus takes the globally defined conditional measure $\mu_{\alpha^{u}(q)}$ on the fiber $\alpha^{u}(q)$ to a measure on the Teichmüller space; the resulting measure $\pi_{*}\left(\mu_{\alpha^{u}(q)}\right)$ is absolutely continuous with respect to the smooth measure m. Furthermore, by Theorem 2.2 the measure $\mathbf{m}$ is also absolutely continuous with respect to $\pi_{*}\left(\mu_{\alpha^{u}(q)}\right)$; indeed, away from the multiple zero locus the mapping $\pi$ is smooth with a smooth inverse, and the multiple zero locus has measure 0 by Theorem 2.2 .

We may therefore consider the corresponding Radon-Nikodym derivative. Introduce a function $\lambda^{+}: \mathcal{Q}^{1} \mathcal{T}_{g} \rightarrow \mathbb{R}$ by the formula

$$
\lambda^{+}(q)=\frac{d \mathbf{m}}{d\left(\pi_{*}\left(\mu_{\alpha^{u}(q)}\right)\right)}(\pi(q)) .
$$

Similarly, we define $\lambda^{-}: \mathcal{Q}^{1} \mathcal{T}_{g} \rightarrow \mathbb{R}$ via the formula

$$
\lambda^{-}(q)=\frac{d \mathbf{m}}{d\left(\pi_{*}\left(\mu_{\alpha^{s}(q)}\right)\right)}(\pi(q)) .
$$

We set

$$
\Lambda(X)=\int_{S(X)} \lambda^{+}(q) d s_{X}(q)=\int_{S(X)} \lambda^{-}(q) d s_{X}(q) .
$$

The equality of the two integrals will be justified by Proposition 2.3. Note that the functions $\lambda^{+}, \lambda^{-}, \Lambda$ are $\Gamma$-invariant. We call $\lambda^{+}, \lambda^{-}$and $\Lambda$ the Hubbard-Masur function.

Note that by the Hubbard-Masur theorem, $\eta^{-}$(or $\eta^{+}$) defines a homeomorphism between the space of all quadratic differentials at $X$ (with arbitrary area) and the space $\mathcal{M F}$. This homeomorphism restricts to a homeomorphism between $S(X)$ and the set

$$
E_{X}=\left\{\beta \in \mathcal{M F}: \operatorname{Ext}_{\beta}(X)=1\right\}
$$

where $\operatorname{Ext}_{\beta}(X)$ is the extremal length at $X$ of the measured foliation $\beta$. Let $\delta_{X}^{ \pm}: E_{X} \rightarrow S(X)$ denote the inverse of $\eta^{ \pm}$.

It is easy to see that the functions $\lambda^{ \pm}$are smooth on the complement of the multiple zero locus.

Proposition 2.3 (Properties of $\left.\lambda^{+}, \lambda^{-}, \Lambda\right)$ Let $X=\pi(q)$.

(i) $\lambda^{+}(q)=\frac{d\left(\delta_{X}^{-}\right)_{*}(\bar{\nu})}{d s_{X}}(q)$, and $\lambda^{-}(q)=\frac{d\left(\delta_{X}^{+}\right)_{*}(\bar{\nu})}{d s_{X}}(q)$.

(ii) $\Lambda(X)=\bar{\nu}\left(\eta^{-}(S(X))\right)=\bar{\nu}\left(\eta^{+}(S(X))\right)$.

(iii) $\Lambda(X)=\bar{\nu}\left(E_{X}\right)=\nu\left(\left\{\beta \in \mathcal{M F}: \operatorname{Ext}_{\beta}(X) \leq 1\right\}\right)$.

(iv) $\Lambda(X)=\bar{\nu}\left(\left\{\beta \in \mathcal{M L}: \operatorname{Ext}_{\beta}(X) \leq 1\right\}\right)$, where $\mathcal{M L}$ is the space of measured laminations.

In (iv), by abuse of notation, $\nu$ denotes Thurston measure on $\mathcal{M L}$ and $\bar{\nu}(E)=\nu($ Cone $(E))$.

Proof. The property (i) follows from the fact that $d \mu=d \mu_{\alpha^{u}} d \mu_{\alpha^{s s}}$, the fact that $\eta_{*}^{-}\left(\alpha^{s s}\right)=\bar{\nu}$, and the fact that if we write for $q \in \mathcal{Q}^{1} \mathcal{T}_{g}, q=(X, v)$ where $X=\pi(q)$ and $v \in S(X)$ then $d \mu(q)=d \mathbf{m}(X) d s_{X}(v)$. The property (ii) follows from (i) after making the change of variable $q \rightarrow \eta^{-}(q)$ in the definition of $\Lambda$. The property (iii) follows from the fact that the image $\eta^{-}(S(X))$ consists exactly of $E_{X}$. Finally (iv) is easily seen to be equivalent to (iii).

The following is proved in 95 ; 
Theorem 2.4 (Boundedness of $\Lambda$ ) There exists a constant $M$ such that $\Lambda(X) \leq M$ for all $X \in$ $\mathcal{T}_{g}$.

For another interpretation of $\Lambda$ in terms of asymptotics of the number of multicurves, see (5.3) below.

\subsubsection{Conformal Densities}

Let the measure $\nu_{X}$ on $\mathcal{P} \mathcal{M F}$ be the image of $\lambda^{-}(q) d s_{X}(q)$ under the identification of $S(X)$ and $\mathcal{P M F}$. That is,

$$
\nu_{X}(U)=\nu\left(\left\{\eta \mid[\eta] \in U, \operatorname{Ext}_{\eta}(X) \leq 1\right\}\right),
$$

where $\nu$ is, as above, Thurston measure on $\mathcal{M F}$, and $[\eta]$ denotes the image of $\eta$ in $\mathcal{P} \mathcal{M F}$. By definition, for $X, Y \in \mathcal{T}_{g}, \xi \in \mathcal{M F}$, we have

$$
\frac{d \nu_{X}}{d \nu_{Y}}([\xi])=\left(\frac{\sqrt{\operatorname{Ext}_{\xi}(Y)}}{\sqrt{\operatorname{Ext}_{\xi}(X)}}\right)^{6 g-6} .
$$

Note that the right-hand side only depends on $[\xi]$.

- Given $[\xi] \in \mathcal{P} \mathcal{M F}$, let $\beta_{\xi}: \mathcal{T}_{g} \times \mathcal{T}_{g} \rightarrow \mathbb{R}$ be the cocycle defined by

$$
\beta_{\xi}(X, Y)=\log \left(\frac{\sqrt{\operatorname{Ext}_{\xi}(X)}}{\sqrt{\operatorname{Ext}_{\xi}(Y)}}\right) .
$$

In our setting $\beta_{\xi}$ plays the role of the Busemann cocycle for the Teichmüller metric, and (formally) the family $\left\{\nu_{X}\right\}_{X \in \mathcal{T}_{g}}$ of finite measures on $P \mathcal{M F}$ is a family of conformal densities of dimension $\delta=6 g-6$ for the cocycle $\beta$; i.e, for any $X, Y \in \mathcal{T}_{g}$ and almost every $\xi \in \mathcal{P} \mathcal{M F}$ we have

$$
\frac{d \nu_{X}}{d \nu_{Y}}([\xi])=\exp \left(\delta \beta_{\xi}(Y, X)\right), \text { and } \nu_{\gamma \cdot X}(\gamma \cdot U)=\nu_{X}(U),
$$

where $U \subset \mathcal{P} \mathcal{M F}$, and $\gamma$ is an element of the mapping class group.

Moreover, by the measure classification result of [LM], $\left\{\nu_{X}\right\}_{X \in \mathcal{T}_{g}}$ is the unique conformal density for the action of the mapping class group on $\mathcal{T}_{g}$ up to scale; the only possibility for $\delta$ is $6 g-6$.

- Given $X \in \mathcal{T}_{g}$, and transverse minimal foliations $\xi, \eta \in \mathcal{M F}$, define

$$
\beta(X,[\xi],[\eta])=\left(\frac{\sqrt{\operatorname{Ext}_{\xi}(X) \operatorname{Ext}_{\eta}(X)}}{A(\xi, \eta)}\right)^{6 g-6},
$$

where $A(\xi, \eta)$ is the area of the quadratic differential with horizontal foliation $\xi$, and vertical foliation $\eta$. Note that the right-hand side only depends on $[\xi],[\eta]$.

Now introduce a measure $\mathbb{P}$ on the product $\mathcal{P} \mathcal{M F} \times \mathcal{P} \mathcal{M F}$ by the formula

$$
\mathbb{P}=\beta(X,[\xi],[\eta]) \nu_{X}([\xi]) \nu_{X}([\eta]) .
$$


Observe that the right-hand side does not depend on $X$ and is invariant under the diagonal action of the mapping class group.

The space $\mathcal{Q}^{1} \mathcal{T}_{g}$ of quadratic differentials fibres over $\mathcal{P} \mathcal{M F} \times \mathcal{P} \mathcal{M F}$ (with fibre $\mathbb{R}$ ) and the measure $\mathbb{P}$ on $\mathcal{P} \mathcal{M F} \times \mathcal{P} \mathcal{M F}$ lifts to (a multiple of) the Lebesgue measure on $\mathcal{Q}^{1} \mathcal{T}_{g}$ (by construction, it is invariant and belongs to the Lebesgue measure class - but this determines the measure uniquely).

A consequence of Theorem 2.9 is that the measures $\nu_{X}$ are the Patterson-Sullivan measures on $\mathcal{T}_{g}$. These constructions are similar to the ones due to Kaimanovich in Ka90a and Ka90b.

\subsection{Measure in Polar Coordinates.}

Recall that $\mathcal{Q}^{1} \mathcal{T}_{g}$ is the space of pairs $(X, q)$ where $X$ is a Riemann surface and $q$ is a holomorphic quadratic differential on $X$. (We occasionally drop $X$ from the notation and denote points of $\mathcal{Q}^{1} \mathcal{T}_{g}$ by $q$ alone).

Fix $X \in \mathcal{T}_{g}$. Then we have a diffeomorphism $\Phi: S(X) \times \mathbb{R}^{+} \rightarrow \mathcal{Q}^{1} \mathcal{T}_{g}$ where $\Phi(q, t)=g_{t} q$ is the point in $\mathcal{Q}^{1} \mathcal{T}_{g}$ which is the endpoint of the length $t$ geodesic segment starting at $X$ and tangent to $q \in S(X)$. We can then write our measure $d \mathbf{m}$ in polar coordinates,

$$
d \mathbf{m}\left(\pi\left(g_{t} q\right)\right)=\Delta(q, t) d s_{X}(q) d t .
$$

Remark: To be completely precise, we should write $d \mathbf{m}\left(\pi\left(g_{t} q\right)\right)=\Phi_{*}\left(\Delta(q, t) d s_{X}(q) d t\right)$, but it is standard practice to avoid this formality with polar coordinates.

Proposition 2.5 Let $K_{1} \supset K$ be compact subsets of $\mathcal{P}(1, \ldots, 1), q \in \mathcal{Q}^{1} \mathcal{T}_{g}$, and suppose $q$ and $g_{t} q$ both belong to $\Gamma K$. Then

$$
|\Delta(q, t)| \leq C e^{h t}
$$

where $C$ depends only on $K$. If in addition

$$
\left|\left\{s \in[0, t]: g_{s} q \in \Gamma K_{1}\right\}\right| \geq(1 / 2) t
$$

then there exists $\alpha>0$ so that

$$
\Delta(q, t)=e^{h t} \lambda^{-}(q) \lambda^{+}\left(g_{t} q\right)+O\left(e^{(h-\alpha) t}\right)
$$

where $\alpha$ and the implied constant on (2.6) depend only on $K_{1}$.

We will prove this proposition in $\$ 3.2$.

\subsection{Mixing.}

Let $\mathcal{U}$ be a open subset of the boundary at infinity of $\mathcal{T}_{g}$ (which can be identified with the space $\mathcal{P} \mathcal{M F}$ of projective measured foliations). For each $X \in \mathcal{T}_{g}$ we may identify $\mathcal{U}$ with a subset $\mathcal{U}(X)$ of $S(X)$. Let $\operatorname{Sect}_{\mathcal{U}}(X) \subset \mathcal{T}_{g}$ denote the set $\bigcup_{t \geq 0} \pi\left(g_{t} \mathcal{U}(X)\right)$, i.e. the sector based at $X$ in the direction $\mathcal{U}$. For a subset $A \subset \mathcal{T}_{g}, \operatorname{Nbhd}_{r}(A)$ denotes the set of points within Teichmüller distance $r$ of $A$. 
Theorem 2.6 For any $X \in \mathcal{T}_{g}$ and any $\epsilon>0$ there exists an open subset $\mathcal{U}$ of $S(X)$ containing the intersection of $S(X)$ with the multiple zero locus such that for any compact $\mathcal{K} \subset \mathcal{T}_{g}$

$$
\limsup _{R \rightarrow \infty} e^{-h R} \mathbf{m}\left(\operatorname{Nbhd}_{1}\left(B_{R}(X) \cap \operatorname{Sect}_{\mathcal{U}}(X) \cap \Gamma \mathcal{K}\right)\right) \leq \epsilon .
$$

Let $\mathcal{K}$ be a compact subset of $\mathcal{T}_{g}$, and let $K^{\prime}$ be a compact subset of the principal stratum $\mathcal{P}(1, \ldots, 1) \subset \mathcal{Q}^{1} \mathcal{T}_{g}$. Let $B_{R}\left(X, \mathcal{K}, K^{\prime}\right)$ denote the set of points $Y \in \Gamma \mathcal{K}$ such that $d(X, Y)<R$, and the geodesic from $X$ to $Y$ spends at least half the time outside $\Gamma K^{\prime}$.

Theorem 2.7 For $\epsilon>0$ and any compact $\mathcal{K} \subset \mathcal{T}_{g}$ there exists compact $K^{\prime} \subset \mathcal{P}(1, \ldots, 1) \subset \mathcal{Q}^{1} \mathcal{T}_{g}$ such that for any $X \in \mathcal{K}$,

$$
\limsup _{R \rightarrow \infty} e^{-h R} \mathbf{m}\left(N b h d_{1}\left(B_{R}\left(X, \mathcal{K}, K^{\prime}\right)\right)\right) \leq \epsilon .
$$

Theorems 2.6 and 2.7 will be proved in 4 .

Let $\psi$ be a nonnegative compactly supported continuous function on $\mathcal{M}_{g}$, which we extend to a function $\psi: \mathcal{Q}^{1} \mathcal{M}_{g} \rightarrow \mathbb{R}$ by making it constant on each sphere $S(X)$. We can consider $\psi$ to be a $\Gamma$-periodic function on $\mathcal{T}_{g}$ (or $\mathcal{Q}^{1} \mathcal{T}_{g}$ ). Let $\phi$ be another such function.

Proposition 2.8 (Mean Ergodic Theorem) We have

$$
\begin{aligned}
& \lim _{R \rightarrow \infty} h e^{-h R} \int_{\mathcal{M}_{g}} \phi(X)\left(\int_{B_{R}(X)} \psi(Y) d \mathbf{m}(Y)\right) d \mathbf{m}(X) \\
= & \frac{1}{\mathbf{m}\left(\mathcal{M}_{g}\right)} \int_{\mathcal{M}_{g}} \phi(X) \Lambda(X) d \mathbf{m}(X) \int_{\mathcal{M}_{g}} \psi(Y) \Lambda(Y) d \mathbf{m}(Y) .
\end{aligned}
$$

Proof. Suppose $\epsilon>0$ is given. Let $\mathcal{K}$ be the union of the supports of $\phi$ and $\psi$. Without loss of generality, we may assume that the support of $\phi$ is small enough so that there exists an open $\mathcal{U} \subset \partial \mathcal{T}_{g}$ such that for each $X$ in the support of $\phi, \mathcal{U}(X)$ contains the intersection of $S(X)$ with the multiple zero locus, and also (2.7) holds. Here, as above, $\mathcal{U}(X)$ is the sector in $S(X)$ corresponding to directions in $\mathcal{U}$.

We break up the integral over $B_{R}(X)$ in (2.9) into two parts: the first over $B_{R}(X) \cap \operatorname{Sect}_{\mathcal{U}}(X) \cap \Gamma \mathcal{K}$ and the second over the complement. The first is bounded by $C_{1} \epsilon$ in view of Theorem 2.7

Let $K^{\prime}$ be as in Theorem 2.7. We use polar coordinates for the integral over $B_{R}(X)$. We get,

$$
\begin{array}{r}
\int_{\mathcal{M}_{g}} \phi(X)\left(\int_{B_{R}(X)} \psi(Y) d \mathbf{m}(Y)\right) d \mathbf{m}(X)= \\
\int_{\mathcal{M}_{g}} \phi(X)\left(\int_{0}^{R} \int_{S(X)} \Delta(q, t) \psi\left(g_{t} q\right) d s_{X}(q) d t\right) d \mathbf{m}(X) .
\end{array}
$$

In view of (2.8), we may assume, (up to error of size $C \epsilon e^{h R}$ ) that $q \in S(X)$ belongs to the compact set $\Gamma K^{\prime \prime}=\bigcup_{X \in \mathcal{K}} S(X) \backslash \mathcal{U}(X)$, which is away from the multiple zero locus. Also note that the left-hand-side of (2.10) is symmetric in $X$ and $Y$ (up to interchanging the functions $\phi$ and $\psi$ ). So we may also assume that $g_{t} q$ also belongs to $\Gamma K^{\prime \prime}$. Also in view of (2.8), we may assume that 
(2.5) holds. (Again the contribution of the part of the integral where this assumption is violated is bounded by $C \epsilon e^{h R}$.)

We now consider the part of the region where none of the three assumptions are violated, i.e. $q \in \Gamma K^{\prime \prime}, g_{t} q \in \Gamma K^{\prime \prime}$ and (2.5) holds. We are finally in a position to use Proposition 2.5 to replace $\Delta(q, t)$ by $e^{h t} \lambda^{-}(q) \lambda^{+}\left(g_{t} q\right)$. Let $\widehat{\lambda^{ \pm}}$denote the truncations of $\lambda^{ \pm}$to $\Gamma K^{\prime \prime}$. We can write

$$
\begin{aligned}
\int_{0}^{R} e^{h t} \int_{\mathcal{M}_{g}} \phi(X) \int_{S(X)} \widehat{\lambda^{-}}(q) \psi\left(g_{t} q\right) \widehat{\lambda^{+}}\left(g_{t} q\right) d s_{X}(q) d \mathbf{m}(X) d t= \\
\qquad \int_{0}^{R} e^{h t} \int_{\mathcal{Q}^{1} \mathcal{M}_{g}} \phi(q) \widehat{\lambda^{-}}(q) \psi\left(g_{t} q\right) \widehat{\lambda^{+}}\left(g_{t} q\right) d \mu(q) d t
\end{aligned}
$$

where we used the fact that $\phi$ is constant on each sphere $S(X)$ and the formula

$$
d \mu(q)=d \mathbf{m}(\pi(q)) d s_{\pi(q)}(q) .
$$

Recall that $\mu\left(\mathcal{Q}^{1} \mathcal{M}_{g}\right)<\infty$ by Theorem 2.1. Now we can apply the mixing property of the geodesic flow to the functions $\phi \widehat{\lambda^{-}}$and $\psi \widehat{\lambda^{+}}$. The right-hand side of (2.11) is then asymptotic to

$$
\begin{aligned}
\frac{e^{h R}}{h \mu\left(\mathcal{Q}^{1} \mathcal{M}_{g}\right)} \int_{\mathcal{Q}^{1} \mathcal{M}_{g}} \phi(q) \widehat{\lambda^{-}}(q) d \mu(q) & \int_{\mathcal{Q}^{1} \mathcal{M}_{g}} \psi(q) \widehat{\lambda^{+}}(q) d \mu(q) \\
& =\frac{e^{h R}}{h \mathbf{m}\left(\mathcal{M}_{g}\right)} \int_{\mathcal{M}_{g}} \phi(X) \Lambda(X) d \mathbf{m}(X) \int_{\mathcal{M}_{g}} \psi(Y) \Lambda(Y) d \mathbf{m}(Y),
\end{aligned}
$$

where the equality holds up to the truncation error. So the right hand side of (2.9) is equal to the left hand side of (2.9) up to an error which is bounded by $C \epsilon$, where $C$ depends only on $\phi$ and $\psi$. Since $\epsilon>0$ is arbitrary, the theorem follows.

The proof of Proposition 2.8 also shows that for any open $\mathcal{U} \subset S(X)$,

$$
\begin{aligned}
\lim _{R \rightarrow \infty} h e^{-h R} \int_{\mathcal{M}_{g}} \phi(X)\left(\int_{B_{R}(X) \cap S e c t \mathcal{U}(X)} \psi(Y) d \mathbf{m}(Y)\right) d \mathbf{m}(X)= \\
\frac{1}{\mathbf{m}\left(\mathcal{M}_{g}\right)} \int_{\mathcal{M}_{g}} \phi(X)\left(\int_{\mathcal{U}} \lambda^{-}(q) d s_{X}(q)\right) d \mathbf{m}(X) \int_{\mathcal{M}_{g}} \psi(Y) \Lambda(Y) d \mathbf{m}(Y) .
\end{aligned}
$$

\subsection{Counting.}

Proof of Theorem 1.2. Let $F_{R}(X, Y)$ denote the cardinality of the intersection of $B_{R}(X)$ with $\Gamma \cdot Y$. Let $\phi$ and $\psi$ be non-negative compactly supported continuous functions on $\mathcal{M}_{g}$. Note that

$$
\int_{\mathcal{M}_{g}} F_{R}(X, Y) \psi(Y) d \mathbf{m}(Y)=\int_{B_{R}(X)} \psi(Y) d \mathbf{m}(Y) .
$$


Hence, by Proposition 2.8 .

$$
\begin{aligned}
\lim _{R \rightarrow \infty} e^{-h R} \int_{\mathcal{M}_{g}} \phi(X) \int_{\mathcal{M}_{g}} F_{R}(X, Y) \psi(Y) d \mathbf{m}(Y) d \mathbf{m}(X) & \\
= & \frac{1}{h \mathbf{m}\left(\mathcal{M}_{g}\right)} \int_{\mathcal{M}_{g}} \phi(X) \Lambda(X) d \mathbf{m}(X) \int_{\mathcal{M}_{g}} \psi(Y) \Lambda(Y) d \mathbf{m}(Y),
\end{aligned}
$$

i.e. $e^{-h R} F_{R}(X, Y)$ converges weakly to $\frac{1}{h \mathbf{m}\left(\mathcal{M}_{g}\right)} \Lambda(X) \Lambda(Y)$. We would like to show that the convergence is pointwise and uniform on compact sets.

Let $\epsilon>0$ be arbitrary. Let $\psi$ be supported on a ball of radius $\epsilon$ around $Y$ (in the Teichmüller distance), and satisfy $\int_{\mathcal{M}_{g}} \psi d \mathbf{m}=1$. Let $\phi$ be supported on a ball of radius $\epsilon$ around $X$, with $\int_{\mathcal{M}_{g}} \phi d \mathbf{m}=1$. By the triangle inequality,

$$
\int_{\mathcal{M}_{g}} \phi(X) \int_{B_{R-3 \epsilon}(X)} \psi(Y) d \mathbf{m}(Y) d \mathbf{m}(X) \leq F_{R}(X, Y) \leq \int_{\mathcal{M}_{g}} \phi(X) \int_{B_{R+3 \epsilon}(X)} \psi(Y) d \mathbf{m}(Y) d \mathbf{m}(X) .
$$

After multiplying both sides by $h e^{-h R}$ and taking $R \rightarrow \infty$, we get, after applying Proposition 2.8 .

$$
\begin{aligned}
& e^{-3 h \epsilon} \frac{1}{\mathbf{m}\left(\mathcal{M}_{g}\right)} \int_{\mathcal{M}_{g}} \phi(X) \Lambda(X) d \mathbf{m}(X) \int_{\mathcal{M}_{g}} \psi(Y) \Lambda(Y) d \mathbf{m}(Y) \leq \liminf _{R \rightarrow \infty} h e^{-h R} F_{R}(X, Y) \leq \\
& \quad \leq \limsup _{R \rightarrow \infty} h e^{-h R} F_{R}(X, Y) \leq e^{3 h \epsilon} \frac{1}{\mathbf{m}\left(\mathcal{M}_{g}\right)} \int_{\mathcal{M}_{g}} \phi(X) \Lambda(X) d \mathbf{m}(X) \int_{\mathcal{M}_{g}} \psi(Y) \Lambda(Y) d \mathbf{m}(Y) .
\end{aligned}
$$

Since $\epsilon>0$ is arbitrary, and $\Lambda$ is uniformly continuous on compact sets, we get

$$
\lim _{R \rightarrow \infty} h e^{-h R} F_{R}(X, Y)=\frac{1}{\mathbf{m}\left(\mathcal{M}_{g}\right)} \Lambda(X) \Lambda(Y)
$$

as required.

In fact, the same argument using (2.12) instead of Proposition 2.8 yields the following:

Theorem 2.9 Suppose $X \in \mathcal{T}_{g}$, and $\mathcal{U}$ is an open subset of the boundary at infinity of $\mathcal{T}_{g}$. Then for any $Y \in \mathcal{T}_{g}$, we have, as $R \rightarrow \infty$,

$$
\left|B_{R}(X) \cap \operatorname{Sect}_{\mathcal{U}}(X) \cap \Gamma \cdot Y\right| \sim \frac{1}{h \mathbf{m}\left(\mathcal{M}_{g}\right)} e^{h R} \Lambda(Y) \int_{\mathcal{U}} \lambda^{-}(q) d s_{X}(q) .
$$

We also obtain the following:

Theorem 2.10 Suppose $X \in \mathcal{T}_{g}$, and $\mathcal{U}$ and $\mathcal{V}$ are open subsets of the boundary at infinity of $\mathcal{T}_{g}$. Then for any $Y \in \mathcal{T}_{g}$, we have, as $R \rightarrow \infty$,

$$
\begin{aligned}
\mid\left\{\gamma \in \Gamma: \gamma \cdot Y \in B_{R}(X) \cap \operatorname{Sect}_{\mathcal{U}}(X)\right. \text { and } & \left.\gamma^{-1} \cdot X \in \operatorname{Sect}_{\mathcal{V}}(Y)\right\} \mid \\
& \sim \frac{1}{h \mathbf{m}\left(\mathcal{M}_{g}\right)} e^{h R} \int_{\mathcal{U}} \lambda^{-}(q) d s_{X}(q) \int_{\mathcal{V}} \lambda^{+}(q) d s_{Y}(q) .
\end{aligned}
$$

The proof of Theorem 2.10 is very similar to that of Theorem 2.9 except that we do not assume that the function $\psi: \mathcal{Q}^{1} \mathcal{M}_{g} \rightarrow \mathbb{R}$ is the pullback under $\pi$ of a function from $\mathcal{M}_{g} \rightarrow \mathbb{R}$. The details are left to the reader. 


\section{The Hodge Norm.}

\subsection{The Hodge Norm for Abelian Differentials.}

\subsubsection{Definition and Basic Properties.}

Let $X$ be a Riemann surface. By definition, $X$ has a complex structure. Let $\mathcal{H}_{X}$ denote the set of holomorphic 1 -forms on $X$. One can define Hodge inner product on $\mathcal{H}_{X}$ by

$$
\langle\omega, \eta\rangle=\int_{X} \omega \wedge \bar{\eta} .
$$

We have a natural map $r: H^{1}(X, \mathbb{R}) \rightarrow \mathcal{H}_{X}$ which sends a cohomology class $\lambda \in H^{1}(X, \mathbb{R})$ to the holomorphic 1-form $r(\lambda) \in \mathcal{H}_{X}$ such that the real part of $r(\lambda)$ (which is a harmonic 1-form) represents $\lambda$. We can thus define the Hodge inner product on $H^{1}(X, \mathbb{R})$ by $\left\langle\lambda_{1}, \lambda_{2}\right\rangle=\left\langle r\left(\lambda_{1}\right), r\left(\lambda_{2}\right)\right\rangle$. We have

$$
\left\langle\lambda_{1}, \lambda_{2}\right\rangle=\int_{X} \lambda_{1} \wedge * \lambda_{2},
$$

where $*$ denotes the Hodge star operator, and we choose harmonic representatives of $\lambda_{1}$ and $* \lambda_{2}$ to evaluate the integral. We denote the associated norm by $\|\cdot\|$. This is the Hodge norm. See [FK].

Let $\alpha$ be a homology class in $H_{1}(X, \mathbb{R})$. We can define the cohomology class $* c_{\alpha} \in H^{1}(X, \mathbb{R})$ so that for all $\omega \in H^{1}(X, \mathbb{R})$,

$$
\int_{\alpha} \omega=\int_{X} \omega \wedge * c_{\alpha}
$$

Then,

$$
\int_{X} * c_{\alpha} \wedge * c_{\beta}=I(\alpha, \beta),
$$

where $I(\cdot, \cdot)$ denotes algebraic intersection number. We have, for any $\omega \in H^{1}(X, \mathbb{R})$,

$$
\left\langle\omega, c_{\alpha}\right\rangle=\int_{X} \omega \wedge * c_{\alpha}=\int_{\alpha} \omega .
$$

We note that $* c_{\alpha}$ is a purely topological construction which depends only on $\alpha$, but $c_{\alpha}$ depends also on the complex structure of $X$.

\subsubsection{The Hodge Norm and the Hyperbolic Metric.}

Let $\alpha$ be a simple close curve on a Riemann surface $X$. Let $\ell_{\alpha}(\sigma)$ denote the length of the geodesic representative of $\alpha$ in the hyperbolic metric which is in the conformal class of $X$.

Fix $\epsilon_{*}>0$ (the Margulis constant) so that any two curves of hyperbolic length less than $\epsilon_{*}$ must be disjoint.

Theorem 3.1 For any constant $D>1$ there exists a constant $c>1$ such that for any simple closed curve $\alpha$ with $\ell_{\alpha}(\sigma)<D$,

$$
\frac{1}{c} \ell_{\alpha}(\sigma)^{1 / 2} \leq\left\|c_{\alpha}\right\|<c \ell_{\alpha}(\sigma)^{1 / 2}
$$

Furthermore, if $\ell_{\alpha}(\sigma)<\epsilon_{0}$ and $\beta$ is the shortest simple closed curve crossing $\alpha$, then

$$
\frac{1}{c} \ell_{\alpha}(\sigma)^{-1 / 2} \leq\left\|c_{\beta}\right\|<c \ell_{\alpha}(\sigma)^{-1 / 2}
$$


Proof. Let $\alpha_{1}, \ldots, \alpha_{n}$ be the curves with hyperbolic length less than $\epsilon_{0}$. For $1 \leq k \leq n$, let $\beta_{k}$ be the shortest curve with $i\left(\alpha_{k}, \beta_{k}\right)=1$, where $i(\cdot, \cdot)$ denotes the geometric intersection number. It is enough to prove (3.1) for the $\alpha_{k}$ and the $\beta_{k}$ (the estimate for other moderate length curves follows from a compactness argument).

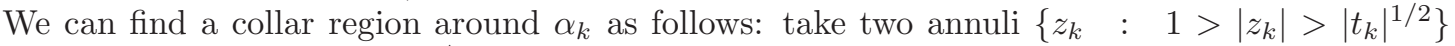
and $\left\{w_{k}: 1>w_{k}>\left|t_{k}\right|^{1 / 2}\right\}$ and identify the inner boundaries via the map $w_{k}=t_{k} / z_{k}$. (This coordinate system is used in e.g. [Fa73, Chapter 3], also [Mas76], [Fo02, Wo03, §3] and elsewhere). The hyperbolic metric $\sigma$ in the collar region is approximately $|d z| /(|z||\log | z||)$. Then $\ell_{\alpha_{k}}(\sigma) \approx 1 /\left|\log t_{k}\right|$. By [Fa73, Chapter 3] any holomorphic 1-form $\omega$ can be written in the collar region as

$$
\left(a_{0}\left(z_{k}+t_{k} / z_{k}, t_{k}\right)+\frac{a_{1}\left(z_{k}+t_{k} / z_{k}, t_{k}\right)}{z_{k}}\right) d z_{k},
$$

where $a_{0}$ and $a_{1}$ are holomorphic in both variables. (We assume here that the limit surface on the boundary of Teichmüller space is fixed). This implies that as $t_{k} \rightarrow 0$,

$$
\omega=\left(\frac{a}{z_{k}}+h\left(z_{k}\right)+O\left(t_{k} / z_{k}^{2}\right)\right) d z_{k}
$$

where $h$ is a holomorphic function which remains bounded as $t_{k} \rightarrow 0$, and the implied constant is bounded as $t_{k} \rightarrow 0$. (Note that when $\left|z_{k}\right| \geq\left|t_{k}\right|^{1 / 2},\left|t_{k} / z_{k}^{2}\right| \leq 1$ ). Now from the condition $\int_{\alpha_{k}} * c_{\beta_{k}}=1$ we see that on the collar of $\alpha_{j}$,

$$
c_{\beta_{k}}+i * c_{\beta_{k}}=\left(\frac{\delta_{k j}}{(2 \pi) z_{j}}+h_{k j}\left(z_{j}\right)+O\left(t_{j} / z_{j}^{2}\right)\right) d z_{j},
$$

where the $h_{k j}$ are holomorphic and bounded as $t_{j} \rightarrow 0$. (We use the notation $\delta_{k j}=1$ if $k=j$ and zero otherwise). Also from the condition $\int_{\beta_{k}} * c_{\alpha_{k}}=1$ we have

$$
c_{\alpha_{k}}+i * c_{\alpha_{k}}=\frac{1}{\left|\log t_{j}\right|}\left(\frac{\delta_{k j}}{z_{j}}+s_{k j}\left(z_{j}\right)+O\left(t_{j} / z_{k}^{2}\right)\right) d z_{j},
$$

where $s_{k j}$ also remains holomorphic and is bounded as $t_{j} \rightarrow 0$. Then,

$$
\left\|c_{\beta_{k}}\right\|^{2}=\left\|* c_{\beta_{k}}\right\|^{2}=\int_{X} c_{\beta_{k}} \wedge * c_{\beta_{k}} \approx \int_{0}^{2 \pi} \int_{t_{k}}^{1} \frac{1}{4 \pi^{2} r^{2}} r d r d \theta \approx \frac{\left|\log t_{k}\right|}{4 \pi}
$$

and

$$
\left\|c_{\alpha_{k}}\right\|^{2}=\left\|* c_{\alpha_{k}}\right\|^{2}=\int_{X} c_{\alpha_{k}} \wedge * c_{\alpha_{k}} \approx \int_{0}^{2 \pi} \int_{t_{k}}^{1} \frac{1}{\left(\log t_{k}\right)^{2} r^{2}} r d r d \theta \approx \frac{2 \pi}{\left|\log t_{k}\right|}
$$

\subsubsection{The Hodge Norm and Extremal Length.}

We recall the following theorem ( $\mathrm{Ac60}$, Bl61): 
Theorem 3.2 (Accola, Blatter) For any Riemann surface $X$ and any $\lambda \in H_{1}(X, \mathbb{R})$,

$$
\left\|c_{\lambda}\right\|^{2}=\sup _{\rho} \inf _{\gamma \in[\lambda]} \frac{\ell_{\gamma}(\rho)^{2}}{\operatorname{Area}(\rho)}
$$

where the supremum is over the metrics $\rho$ which are in the conformal class of $X$, and the infimum is over all the multicurves $\gamma$ homologous to $\lambda$.

Note that in the definition (2.1) of extremal length, the infimum is is over all the simple closed curves $\gamma$ homotopic to $\lambda$. Recall that the extremal metric $\rho$ is always the flat metric obtained from a quadratic differential $q$, and in this metric the entire surface consists of a flat cylinder in which $\lambda$ is the core curve. Thus, if this quadratic differential $q$ is in fact the square of an Abelian differential then it follows from Theorem 3.2 that

$$
\operatorname{Ext}_{\lambda}(X)=\left\|c_{\lambda}\right\|^{2}
$$

\subsubsection{The Hodge Norm and the Geodesic Flow.}

Let $\Omega^{1} \mathcal{T}_{g}$ denote the space of (unit area) holomorphic 1-forms on surfaces of genus $g$. Recall that $g_{t}$, the Teichmüller geodesic flow, preserves $\Omega^{1} \mathcal{T}_{g}$ (where we map $\Omega^{1} \mathcal{T}_{g}$ into $\mathcal{Q}^{1} \mathcal{T}_{g}$ by squaring abelian differentials). Fix a point $S$ in $\Omega^{1} \mathcal{T}_{g}$; then $S$ is a pair $(X, \omega)$ where $\omega$ is a holomorphic 1-form on $X$. Let $\|\cdot\|_{0}$ denotes the Hodge norm on the surface $X_{0}=X$, and let $\|\cdot\|_{t}$ denote the Hodge norm on the surface $X_{t}=\pi\left(g_{t} S\right)$.

The following fundamental result is due to Forni [Fo02, §2]:

Theorem 3.3 For any $\theta \in H^{1}(X, \mathbb{R})$ and any $t \geq 0$,

$$
\|\theta\|_{t} \leq e^{t}\|\theta\|_{0} .
$$

Moreover, there exists a constant $C$ depending only on the genus, such that if $\langle\theta, \omega\rangle=0$, and for some compact subset $\mathcal{K}$ of $\mathcal{M}_{g}$, the geodesic segment $\left[S, g_{t} S\right]$ spends at least half the time in $\pi^{-1}(\mathcal{K})$, then we have

$$
\|\theta\|_{t} \leq C e^{(1-\alpha) t}\|\theta\|_{0}
$$

where $\alpha>0$ depends only on $\mathcal{K}$.

\subsection{Proof of Proposition 2.5.}

In this section we prove Proposition 2.5. We recall the setting: given $X \in \mathcal{T}_{g}$, let $g_{t} q$ be the point in $\mathcal{Q}^{1} \mathcal{T}_{g}$ which is the endpoint of the length $t$ geodesic segment starting at $X$ and tangent to $q \in S(X)$. $\Delta(q, t)$ is then given by:

$$
d \mathbf{m}\left(\pi\left(g_{t} q\right)\right)=\Delta(q, t) d s_{X}(q) d t .
$$

Let $K_{1} \supset K$ be compact subsets of $\mathcal{P}(1, \ldots, 1), q \in \mathcal{Q}^{1} \mathcal{T}_{g}$, and suppose $q$ and $g_{t} q$ both belong to $\Gamma K$. Proposition 2.5 asserts a general bound, inequality (2.4):

$$
|\Delta(q, t)| \leq C e^{h t}
$$


where $C$ depends only on $K$. If we are given some more information about the trajectory $\left\{g_{s} q\right\}_{0 \leq s \leq t}$, in particular that it spends a portion of its time in a compact set, we can say more. Precisely, if the inequality (2.5):

$$
\left|\left\{s \in[0, t]: g_{s} q \in \Gamma K_{1}\right\}\right| \geq(1 / 2) t
$$

is satisfied then there exists $\alpha>0$ so that the inequality (2.6):

$$
\Delta(q, t)=e^{h t} \lambda^{-}(q) \lambda^{+}\left(g_{t} q\right)+O\left(e^{(h-\alpha) t}\right)
$$

holds. Moreover, $\alpha$ and the implied constant in (2.6) depend only on $K_{1}$.

Proof. Without loss of generality, $q \in K$. Let $X=\pi(q), Y=\pi\left(g_{t} q\right)$. Write $Y=\gamma z$ where $\gamma \in \Gamma$ and $z \in K$. Let $A$ be the matrix of $\gamma$ acting on the odd part of the homology of the orienting double cover $\tilde{X}$ of $q$ (i.e. $A$ is the Kontsevitch-Zorich cocycle.) Let $\langle\cdot, \cdot\rangle$ denote the Hodge inner product, and $A^{*}$ denote the adjoint of $A$, where we view $A$ as a map between the inner product spaces determined by the Hodge inner product at $X$ and $Y$ respectively. Let $n=h=\operatorname{dim} H_{\text {odd }}^{1}(\tilde{X}, \mathbb{R})$, and let $e_{1}, \ldots e_{n}$ be an orthonormal basis for $H_{\text {odd }}^{1}(\tilde{X}, \mathbb{R})$ with respect to $\langle\cdot, \cdot\rangle$, so that

$$
A^{*} A e_{i}=\lambda_{i}^{2} e_{i},
$$

where $e^{t}=\lambda_{1} \geq \cdots \geq \lambda_{n}=e^{-t}>0$. Then $\left\|A e_{i}\right\|=\lambda_{i}$. Note that $e_{1}$ is the imaginary part of the holomorphic 1-form $\tilde{\omega}$ on $\tilde{X}$ for which $\tilde{\omega}^{2}=q$, and $e_{n}$ is the real part of $\tilde{\omega}$. We can read off that $\lambda_{1}=e^{t}, \lambda_{n}=e^{-t}$. Also since $A$ is symplectic, $\lambda_{2}=\lambda_{n-1}^{-1}$.

Note since we are assuming $q \in K$, the pair $(\tilde{X}, \tilde{\omega})$ associated to $q$ belongs to a compact subset (depending only on $K$ ) of the space of Abelian differentials. Then, by Theorem 3.3 ,

$$
\lambda_{2}=O\left(e^{t}\right) \text { and } \lambda_{n-1}^{-1}=O\left(e^{t}\right),
$$

and if (2.5) holds,

$$
\lambda_{2}=O\left(e^{(1-\alpha) t}\right) \text { and } \lambda_{n-1}^{-1}=O\left(e^{(1-\alpha) t}\right) .
$$

Since $A$ has determinant 1 , the product of the $\lambda_{i}$ is 1 .

We can identify the tangent space to $\mathcal{Q} \mathcal{T}_{g}$ with $H_{\text {odd }}^{1}\left(\tilde{X}, \mathbb{R}^{2}\right)=H_{\text {odd }}^{1}(\tilde{X}, \mathbb{R}) \otimes \mathbb{R}^{2}$.

Let $e_{i}^{+}=e_{i} \otimes\left(\begin{array}{l}1 \\ 0\end{array}\right)$ and $e_{i}^{-}=e_{i} \otimes\left(\begin{array}{l}0 \\ 1\end{array}\right)$ so that the $e_{i}^{+}$and $e_{i}^{-}$together form a basis for the tangent space to $\mathcal{Q} \mathcal{T}_{g}$ at $q$. Then $\left(d g_{t}\right)_{*}\left(e_{i}^{+}\right)=e^{t} A e_{i}^{+}$, and $\left(d g_{t}\right)_{*}\left(e_{i}^{-}\right)=e^{-t} A e_{i}^{-}$. (In the above we adopt the convention that $A$ acts on the tensor product $H_{\text {odd }}^{1}(\tilde{X}, \mathbb{R}) \otimes \mathbb{R}^{2}$ by acting on the $H_{\text {odd }}^{1}(\tilde{X}, \mathbb{R})$ factor, and trivially on the second factor.)

Let $u_{1}=e_{1}^{+}-e_{n}^{-}$. We can complete the set $\left\{u_{1}\right\}$ to a basis $\left\{u_{1}, \ldots, u_{n-1}\right\}$ for the tangent space of $\mathcal{Q}^{1} \mathcal{T}_{g}$ at $q$, so that for $2 \leq i \leq n-1, u_{i}=e_{i}^{+}+w_{i}$, where $w_{i}$ is in the span of $\left\{e_{2}^{-}, \ldots, e_{n-1}^{-}\right\}$. For consistency, we let $w_{1}=-e_{n}^{-}$. Note that the $\left\|w_{i}\right\|$ are bounded since $q$ belongs to a compact set $K$, and the leaves of $\mathcal{F}^{u}$ are transverse to the spheres $S(X)$ on $K$.

We extend the Hodge inner product $\langle\cdot, \cdot\rangle$ to $H_{\text {odd }}^{1}\left(\tilde{X}, \mathbb{R}^{2}\right)$ by declaring that $\left\langle e_{i}^{+}, e_{j}^{-}\right\rangle=0$ for all 
$i, j$. This also extends the Hodge norm. We now estimate

$$
\begin{aligned}
\left\|\left(d g_{t}\right)_{*}\left(u_{1} \wedge \cdots \wedge u_{n-1}\right)\right\|^{2} & =\left\langle u_{1} \wedge \cdots \wedge u_{n-1},\left[\left(d g_{t}\right)_{*}\right]^{*}\left[\left(d g_{t}\right)_{*}\right] u_{1} \wedge \cdots \wedge u_{n-1}\right\rangle \\
& =\sum_{P}\left\langle u_{1} \wedge \cdots \wedge u_{n-1},\left[\left(d g_{t}\right)_{*}\right]^{*}\left[\left(d g_{t}\right)_{*}\right]\left(\bigwedge_{i \notin P} e_{i}^{+} \wedge \bigwedge_{i \in P} w_{i}\right)\right\rangle \\
& =\sum_{P}\left\langle u_{1} \wedge \cdots \wedge u_{n-1},\left(\bigwedge_{i \notin P} e^{2 t} \lambda_{i}^{2} e_{i}^{+} \wedge \bigwedge_{i \in P} e^{-2 t} A^{*} A w_{i}\right)\right\rangle
\end{aligned}
$$

where $\left[\left(d g_{t}\right)_{*}\right]^{*}$ is the adjoint of the linear transformation $\left(d g_{t}\right)_{*}$, and the sum is over all subsets $P$ of $\{1, \ldots, n-1\}$.

Note that by (3.5), for $1 \leq i \leq n-1$

$$
\left\|e^{-2 t} A^{*} A w_{i}\right\| \leq C \leq C\left\|e^{2 t} \lambda_{i}^{2}\right\| .
$$

Thus all the terms in (3.7) are bounded by $C e^{2 t(n-1)} \lambda_{1}^{2} \prod_{i=2}^{n-1} \lambda_{i}^{2}=C e^{2 n t}=C e^{2 h t}$. Thus,

$$
\left\|\left(d g_{t}\right)_{*}\left(u_{1} \wedge \cdots \wedge u_{n-1}\right)\right\|=O\left(e^{h t}\right) .
$$

By definition, $\Delta(q, t)$ is the determinant of the linear transformation $D=\left(d \pi_{Y}\right)_{*}\left(d g_{t}\right)_{*}$. Since $Y$ is in a compact set, the norm of $\left(d \pi_{Y}\right)_{*}$ is bounded. This together with (3.8) implies (2.4). If we assume (3.6), we get for any $1 \leq i \leq n-1$,

$$
\left\|e^{-2 t} A^{*} A w_{i}\right\| \leq C e^{-2 \alpha t} \leq C e^{-2 \alpha t} e^{2 t} \lambda_{i}^{2},
$$

which implies that the contribution of all the terms in (3.7) to $\Delta(q, t)$ except for the term with $P=\emptyset$ is $O\left(e^{(h-\alpha) t}\right)$. It remains to evaluate the term with $P=\emptyset$. Dropping the rest of the terms is equivalent to replacing the map $\Phi(q, t)$ by the composition of three maps:

1. Projecting from the sphere $S(X)$ to the leaf of the expanding foliation $\mathcal{F}^{u}$ passing through $q$.

2. Flowing by time $t$.

3. Applying the map $\pi_{Y}$ to project from the leaf of $\mathcal{F}^{u}$ through $g_{t} q$ to Teichmüller space $\mathcal{T}_{g}$.

The Jacobian of the resulting map is the product of the Jacobians from steps 1), 2) and 3). Step 1 ) gives a factor of $\lambda^{-}(q)$, step 2) gives a factor of $e^{h t}$, and step 3) gives a factor of $\lambda^{+}\left(g_{t} q\right)$, so one gets formula (2.6).

\subsection{The Hodge Norm and Quadratic Differentials.}

\subsubsection{The Hodge Norm and the Flat Metric.}

Theorem 3.1 allows us to estimate the Hodge norm in terms of the hyperbolic metric. In this subsection we state a lemma which allows us to estimate the Hodge norm in terms of the flat metric. This is done by first estimating the hyperbolic metric in terms of the flat metric, and then using Theorem 3.1 .

Let $q$ be a holomorphic quadratic differential, and let $\ell_{q}$ denote length in the flat metric defined by $q$. For each $\epsilon>0$ let $K(\epsilon)$ denote the complex generated by all saddle connections shorter 
then $\epsilon$ (see [EM01, $\S 6]$ ). Recall that $K(\epsilon)$ contains all saddle connections shorter then $\epsilon$, and that $\ell_{q}(\partial K(\epsilon))=O(\epsilon)$, where the implied constant depends only on the genus. (Also if $\mathcal{C}$ is a cylinder whose boundary is shorter then $\epsilon$, we include $\mathcal{C}$ in $K(\epsilon))$. Let $\epsilon_{1}<\cdots<\epsilon_{n}$ denote the values of $\epsilon$ where $K(\epsilon)$ changes. (Note that $n$ is bounded in terms of the genus). Pick a constant $C \gg 1$ and drop all $\epsilon_{i}$ such that $\epsilon_{i}>\epsilon_{i+1} / C$. After renumbering, we obtain new $\epsilon_{i} 1 \leq i \leq m$, with $\epsilon_{i}<\epsilon_{i+1} / C$. To simplify notation, we let $\epsilon_{m+1}=1$. For $1 \leq i \leq m$, let $K_{i}$ denote the complex $K\left(\epsilon_{i}\right)$.

Lemma 3.4 (Rafi, Ra05]) Let $q$ be a holomorphic quadratic differential on a surface $\Sigma_{g}$, and let $\sigma$ be the hyperbolic metric on $\Sigma_{g}$ in the same conformal class as $q$. Choose $C \gg 1$, and let the $\epsilon_{i}$, $1 \leq i \leq m$ and the complexes $K_{i}$ be as in the previous paragraph. Then

(a) There exists a constant $\epsilon_{0}$ depending only on $C$ and the genus (with $\epsilon_{0} \rightarrow 0$ as $C \rightarrow \infty$ ) such that any simple closed curve $\alpha$ on $\Sigma_{g}$ with the hyperbolic length $\ell_{\alpha}(\sigma)<\epsilon_{0}$ is homotopic to a connected component of the boundary of one of the $K_{i}$.

(b) Let $\gamma$ be a connected component of $\partial K_{i}$. If $\gamma$ is not a core curve of a flat annulus, then $\ell_{\gamma}(\sigma) \geq C_{1} /\left|\log \epsilon_{i}\right|$, where $C_{1}$ is a constant depending only on the genus.

\subsubsection{The Modified Hodge Norm and Quadratic Differentials.}

Theorem 3.3 gives a partial hyperbolicity property of the geodesic flow on spaces of Abelian differentials. In our applications, we need a similar property for the spaces $\mathcal{Q}^{1} \mathcal{T}_{g}$ of quadratic differentials. A standard construction, given $X \in \mathcal{T}_{g}$ and $q$ a holomorphic quadratic differential on $X$, is to pass to the possibly ramified double cover on which the foliation defined by $q$ is orientable. This yields a surface $\tilde{X}$ with an holomorphic Abelian differential $\omega$. However, a major difficulty is the following: even if $q$ belongs to a compact subset of $\mathcal{Q}^{1} \mathcal{T}_{g}$, the complex structure on $\tilde{X}$ may have very short closed curves in the hyperbolic metric. This may occur since even if one restricts $q$ to compact subsets of Teichmüller space, the flat structure defined by $q$ may have arbitrarily short saddle connections (connecting distinct zeroes). Such a saddle connection may lift to a very short loop when one takes a double cover. Other more complicated types of degeneration are also possible. However, the following simple observation is key to our approach:

Lemma 3.5 For every compact subset $\mathcal{K} \subset \mathcal{M}_{g}$ there exists a constant $\epsilon_{0}>0$ depending only on $\mathcal{K}$ such that for any $q \in \pi^{-1}(\mathcal{K})$, the flat structure associated to the holomorphic quadratic differential on the orienting cover of $q$ has no closed trajectories of Euclidean length less than $\epsilon_{0}$ which are part of a flat cylinder.

Together with Lemma 3.4 (b), Lemma 3.5 will allow us to estimate the hyperbolic length of short curves on $\tilde{X}$.

Proof of Lemma 3.5. Given the set $\mathcal{K}$, there exists a constant $\epsilon_{0}>0$ such that any loop of length less than $\epsilon_{0}$ in the flat metric is contractible. Now suppose $q \in \pi^{-1}(\mathcal{K})$, and $\gamma$ is a trajectory on the associated orienting double cover which has length less than $\epsilon_{0}$, and is part of a flat cylinder $C$. Let $\gamma_{1}$ be the projection of $\gamma$ to the original flat structure defined by $q$. Then $\gamma_{1}$ must also be a part of a flat cylinder, and the length of $\gamma_{1}$ must be at most $\epsilon_{0}$. Then, from the definition of $\epsilon_{0}$ it follows that $\gamma_{1}$ must be contractible. But this is impossible since the curvature of $\gamma_{1}$ is zero, and we are not allowing $q$ to have poles. 
Short bases. Suppose $(X, \omega) \in \Omega^{1} \mathcal{T}_{g}$ (where the notation $\Omega^{1} \mathcal{T}_{g}$ is defined in 3.1.4). Fix $\epsilon_{1}<\epsilon_{*}$ (where $\epsilon_{*}$ is the Margulis constant defined in 3.1.2) and let $\alpha_{1}, \ldots, \alpha_{k}$ be the curves with hyperbolic length less than $\epsilon_{1}$ on $X$. For $1 \leq i \leq k$, let $\beta_{i}$ be the shortest curve in the flat metric defined by $\omega$ with $i\left(\alpha_{i}, \beta_{i}\right)=1$. Let $\gamma_{r}, 1 \leq r \leq 2 g-2 k$ be moderate length curves on $X$ so that the $\alpha_{j}, \beta_{j}$ and $\gamma_{j}$ are a symplectic basis $\mathcal{S}$ for $H_{1}(X, \mathbb{R})$. We will call such a basis short.

The functions $\chi_{i}(\omega)$ and the modified Hodge norm. We would like to use the Hodge norm on double covers of surfaces in $\mathcal{Q}^{1} \mathcal{T}_{g}$. One difficulty is that if one takes the double cover of some quadratic differential in the multiple zero locus, the Hodge norm in some directions tangent to the multiple zero locus might vanish. As a consequence, if we use the Hodge norm to define a "Hodge distance" on $\mathcal{Q}^{1} \mathcal{T}_{g}$, then we find that some balls are not compact, and the resulting "distance" does not separate points on the multiple zero locus.

We now define a modification of the Hodge norm in order to avoid these problems. The modified norm is defined on the tangent space to the space of pairs $(X, \omega)$ where $X$ is a Riemann surface and $\omega$ is a holomorphic 1-form on $X$. Unlike the Hodge norm, the modified Hodge norm will depend not only on the complex structure on $X$ but also on the choice of a holomorphic 1-form $\omega$ on $X$. Let $\left\{\alpha_{i}, \beta_{i}, \gamma_{r}\right\}_{1 \leq i \leq k, 1 \leq r \leq 2 g-2 k}$ be a short basis for $(X, \omega)$. For $1 \leq i \leq k$ we define $\chi_{i}(\omega)$ to be 0 if $\alpha_{i}$ has a flat annulus in the flat metric defined by $\omega$ and 1 otherwise.

We can write any $\theta \in H^{1}(X, \mathbb{R})$ as

$$
\theta=\sum_{i=1}^{k} a_{i}\left(* c_{\alpha_{i}}\right)+\sum_{i=1}^{k} b_{i} \ell_{\alpha_{i}}(\sigma)^{1 / 2}\left(* c_{\beta_{i}}\right)+\sum_{r=1}^{2 g-2 k} u_{r}\left(* c_{\gamma_{r}}\right) .
$$

(Recall that $\sigma$ denotes the hyperbolic metric in the conformal class of $X$, and for a curve $\alpha$ on $X$, $\ell_{\alpha}(\sigma)$ denotes the length of $\alpha$ in the metric $\left.\sigma\right)$. We then define

$$
\|\theta\|^{\prime}=\|\theta\|+\left(\sum_{i=1}^{k} \chi_{i}(\omega)\left|a_{i}\right|+\sum_{i=1}^{k}\left|b_{i}\right|+\sum_{r=1}^{2 g-2 k}\left|u_{r}\right|\right) .
$$

From (3.10) we have for $1 \leq i \leq k$,

$$
\left\|* c_{\alpha_{i}}\right\|^{\prime} \approx 1,
$$

as long as $\alpha_{i}$ has no flat annulus in the metric defined by $\omega$. Similarly, from (3.2) we have

$$
\left\|* c_{\beta_{i}}\right\|^{\prime} \approx\left\|* c_{\beta_{i}}\right\| \approx \frac{1}{\ell_{\alpha_{i}}(\sigma)^{1 / 2}} .
$$

In addition, in view of Theorem 3.1, if $\gamma$ is any other moderate length curve on $X,\left\|* c_{\gamma}\right\|^{\prime} \approx\left\|* c_{\gamma}\right\|=$ $O(1)$. Thus, if $\mathcal{B}$ is a short basis associated to $\omega$, then for any $\gamma \in \mathcal{B}$,

$$
\operatorname{Ext}_{\gamma}(\omega)^{1 / 2} \approx\left\|* c_{\gamma}\right\| \leq\left\|* c_{\gamma}\right\|^{\prime}
$$

(cf. (3.4)). (By $\operatorname{Ext}_{\gamma}(\omega)$ we mean the extremal length of $\gamma$ in $X$, the conformal structure defined by $\omega)$.

Remark. From the construction, we see that the modified Hodge norm is greater than the Hodge norm. Also, if the flat length of shortest curve in the flat metric defined by $\omega$ is greater than $\epsilon_{1}$, then for any cohomology class $\lambda$, for some $C$ depending on $\epsilon_{1}$ and the genus,

$$
\|\lambda\|^{\prime} \leq C\|\lambda\|
$$

i.e. the modified Hodge norm is within a multiplicative constant of the Hodge norm. 


\subsection{Non-expansion of the modified Hodge norm.}

In this subsection we will prove a weak version of Theorem 3.3 for the modified Hodge norm.

Let $\|\cdot\|_{0}^{\prime}$ denote the modified Hodge norm on the surface $S_{0}=S=\left(X_{0}, \omega_{0}\right)$, and let $\|\cdot\|_{t}^{\prime}$ denote the modified Hodge norm on the surface $S_{t}=g_{t} S=\left(X_{t}, \omega_{t}\right)$.

Theorem 3.6 There exists a constant $C$ depending only on the genus and on $\epsilon_{1}$, such that for any $\theta \in H^{1}(X, \mathbb{R})$ and any $t \geq 0$,

$$
\|\theta\|_{t}^{\prime} \leq C e^{t}\|\theta\|_{0}^{\prime}
$$

The proof of Theorem 3.6 is based on the following lemma:

Lemma 3.7 (Rafi) Suppose $\left(X_{0}, \omega_{0}\right) \in \Omega^{1} \mathcal{T}_{g}$, and write $\left(X_{t}, \omega_{t}\right)$ for $g_{t}\left(X_{0}, \omega_{0}\right)$. Suppose $\mathcal{B}$ is a short basis in the flat structure defined by $\omega_{0}$, and $\mathcal{B}^{\prime}$ is a short basis in the flat structure defined by $\omega_{t}$. Suppose $\alpha$ is a curve of hyperbolic length $<\epsilon_{1}$ on both $X_{0}$ and $X_{t}$ (so that in particular $\alpha \in \mathcal{B}$ and $\left.\alpha \in \mathcal{B}^{\prime}\right)$, and that $\alpha$ has no flat annulus. Let $\beta^{\prime} \in \mathcal{B}^{\prime}$ denote the curve with $i\left(\alpha, \beta^{\prime}\right)=1$. Then, for any $\gamma \in \mathcal{B}$, we have

$$
i\left(\gamma, \beta^{\prime}\right) \leq C e^{t} \operatorname{Ext}_{\gamma}\left(X_{0}\right)^{1 / 2},
$$

where $i(\cdot, \cdot)$ denotes the intersection number.

Recall that given simple closed curves $\alpha$ and $\beta$ on $\Sigma_{g}$, the intersection number $i(\alpha, \beta)$ is the minimum number of points in which representatives of $\alpha$ and $\beta$ must intersect.

Proof of Lemma 3.7. First we claim that $i\left(\gamma, \beta^{\prime}\right) \leq i(\gamma, P)$, where $P$ is the shortest pants decomposition of $X_{t}$. This is because, either $\gamma$ is disjoint from $\alpha$ or if it intersects $\alpha$, the relative twisting of $\gamma$ and $\beta^{\prime}$ is bounded (see $\left.\underline{\operatorname{Ra} 07}, \S 4\right]$ ). But this shortest pants decomposition has extremal length less than a constant times $e^{2 t}$ in $X_{0}$. Using [Mi93, Lemma 5.1] we have

$$
i(\gamma, P)^{2} \leq \operatorname{Ext}_{\gamma}\left(X_{0}\right) \operatorname{Ext}_{P}\left(X_{0}\right) \leq C^{2} e^{2 t} \operatorname{Ext}_{\gamma}\left(X_{0}\right) .
$$

This finishes the proof.

Proof of Theorem 3.6. Let $\alpha_{1}^{\prime}, \ldots, \alpha_{k}^{\prime}$ be the curves with hyperbolic length less than $\epsilon_{1}$ on $X_{t}=$ $\pi\left(S_{t}\right)$. Let $\beta_{1}^{\prime}, \ldots \beta_{k}^{\prime}$ be the shortest curves in the Euclidean metric on $S_{t}$ such that $i\left(\alpha_{r}, \beta_{j}\right)=\delta_{r j}$. Let $\gamma_{r}^{\prime}, 1 \leq r \leq 2 g-k$ be moderate length curves on $X_{t}$ so that the $\alpha_{j}, \beta_{j}^{\prime}$ and $\gamma_{j}^{\prime}$ are a symplectic basis $\mathcal{S}^{\prime}$ for $H_{1}(X, \mathbb{R})$. Thus $\mathcal{S}^{\prime}$ is a short basis. For any $\theta \in H^{1}(X, \mathbb{R})$, we may write

$$
\theta=\sum_{i=1}^{k} a_{i}\left(* c_{\alpha_{i}^{\prime}}\right)+\sum_{i=1}^{k} b_{i} \ell_{\alpha_{i}^{\prime}}(\sigma)^{1 / 2}\left(* c_{\beta_{i}^{\prime}}\right)+\sum_{r=1}^{2 g-k} u_{i}\left(* c_{\gamma_{r}^{\prime}}\right) .
$$

In view of (3.10) and Theorem 3.3 it is enough to show that

$$
\sum_{i=1}^{k} \chi_{i}\left(\omega_{t}\right)\left|a_{i}\right|+\sum_{i=1}^{k}\left|b_{i}\right|+\sum_{r=1}^{2 g-k}\left|u_{r}\right| \leq C e^{t}\|\theta\|_{0}^{\prime} .
$$

We have

$$
b_{i}=\frac{1}{\ell_{\alpha_{i}^{\prime}}(\sigma)^{1 / 2}}\left\langle c_{\alpha_{i}^{\prime}}, \theta\right\rangle_{t} \leq \frac{1}{\ell_{\alpha_{i}^{\prime}}(\sigma)^{1 / 2}}\left\|c_{\alpha_{i}^{\prime}}\right\|_{t}\|\theta\|_{t} \leq\|\theta\|_{t} \leq C e^{t}\|\theta\|_{0} \leq C e^{t}\|\theta\|_{0}^{\prime} .
$$


Here, $\langle\cdot, \cdot\rangle_{t}$ denotes the Hodge inner product on $X_{t}$. Also let $\delta_{i}^{\prime} \in \mathcal{S}^{\prime}$ be the element with $i\left(\gamma_{i}^{\prime}, \delta_{i}^{\prime}\right)=1$. Then,

$$
\left|u_{i}\right|=\left\langle c_{\delta_{i}^{\prime}}, \theta\right\rangle_{t} \leq\left\|c_{\delta_{i}^{\prime}}\right\|_{t}\|\theta\|_{t} \leq C_{1}\|\theta\|_{t} \leq C_{2} e^{t}\|\theta\|_{0} \leq C_{3} e^{t}\|\theta\|_{0}^{\prime},
$$

where $C_{1}, C_{2}$ and $C_{3}$ depend only on the genus. It remains to bound $\left|a_{i}\right|$. We may assume that $\alpha_{i}$ has length less than $\epsilon_{1}$ on $X_{0}$ as well. (If $\alpha_{i}$ is longer then $\epsilon_{1}$ on $X_{0}$, we can put in an intermediate point $X_{0}^{\prime}$ where $\alpha_{i}$ has length $\approx \epsilon_{1}$.)

Let $\mathcal{S}=\left\{\alpha_{i}, \beta_{i}, \gamma_{i}\right\}$ be a short basis for $S_{0}$. It is enough to prove (3.17) assuming $\theta=* c_{\lambda}$ for some $\lambda \in \mathcal{S}$. Then,

$$
a_{i}=\left\langle * c_{\beta_{i}^{\prime}}, c_{\lambda}\right\rangle=I\left(\lambda, \beta_{i}^{\prime}\right) .
$$

Since the algebraic intersection number $I(\cdot, \cdot)$ is bounded by the geometric intersection number $i(\cdot, \cdot)$, we have

$$
\left|a_{i}\right|=\left|I\left(\lambda, \beta_{i}^{\prime}\right)\right| \leq i\left(\lambda, \beta_{i}^{\prime}\right) \leq C e^{t} \operatorname{Ext}_{\lambda}\left(X_{0}\right)^{1 / 2} \leq C e^{t}\left\|* c_{\lambda}\right\|^{\prime},
$$

where we have used Lemma 3.7 and 3.13).

\subsection{The Euclidean, Teichmüller and Hodge Distances.}

Note that we can locally identify a leaf of $\mathcal{F}^{s}\left(\right.$ or $\left.\mathcal{F}^{u}\right)$ with a subspace of $H^{1}(\tilde{X}, \mathbb{R})$, where $\tilde{X}$ is the double cover of $X \in \mathcal{T}_{g}$. If $\gamma$ is a map from $[0, r]$ into some leaf of $\mathcal{F}^{s s}$, then we define the (modified) Hodge length $\ell(\gamma)$ of $\gamma$ as $\int_{0}^{r}\left\|\gamma^{\prime}(t)\right\|^{\prime} d t$, where $\|\cdot\|^{\prime}$ is the modified Hodge norm. If $q$ and $q^{\prime}$ belong to the same leaf of $\mathcal{F}^{s s}$, then we define the Hodge distance $d_{H}\left(q, q^{\prime}\right)$ to be the infimum of $\ell(\gamma)$ where $\gamma$ varies over paths connecting $q$ and $q^{\prime}$ and staying in the leaf of $\mathcal{F}^{s s}$ containing $q$ and $q^{\prime}$. We make the same definition if $q$ and $q^{\prime}$ are on the same leaf of $\mathcal{F}^{u u}$.

In what follows $\mathcal{K}$ is a compact subset of $\mathcal{T}_{g}$, and all implied constants depend on $\mathcal{K}$. Given the set $\mathcal{K}$, there exists a constant $\epsilon_{0}>0$ such that for any $q \in \pi^{-1}(\mathcal{K})$ any loop of length less than $\epsilon_{0}$ in the flat metric given by $q$ is contractible.

For each $q \in \pi^{-1}(\mathcal{K})$ there exists a canonical (marked) Delaunay triangulation of the flat metric given by $q$ (see MasSm91, §4]). Since there are only finitely many combinatorial types of (unmarked) triangulations on a genus $g$ surface, and the mapping class group $\Gamma$ acts properly discontinuously, there are only finitely many combinatorial types $\alpha$ of marked Delaunay triangulations on $\pi^{-1}(\mathcal{K})$. Call this set $I$. We have

$$
\pi^{-1}(\mathcal{K})=\bigsqcup_{\alpha \in I} W_{\alpha}
$$

where $W_{\alpha}$ is the subset of $\pi^{-1}(\mathcal{K})$ where the combinatorial type of the marked Delaunay triangulation is given by $\alpha$. Let $J \subset I$ denote the subset such that for $\alpha \in J, W_{\alpha}$ is relatively open in $\pi^{-1}(\mathcal{K})$. Then we may write

$$
\pi^{-1}(\mathcal{K})=\bigsqcup_{\alpha \in J} W_{\alpha}^{\prime}
$$

where $W_{\alpha} \subseteq W_{\alpha}^{\prime} \subseteq \overline{W_{\alpha}}$. If we choose a basis $\mathcal{S}$ for $H_{1}^{\text {odd }}(\tilde{X}, \mathbb{Z})$, then we have a period map $\Phi_{\mathcal{S}}: \mathcal{Q}^{1} \mathcal{T}_{g} \rightarrow \mathbb{C}^{h}$ given by integrating the canonical square root of $q$ along the chosen basis. Recall that this is a local coordinate system for $\mathcal{Q}^{1} \mathcal{T}_{g}$. In fact the following holds:

Lemma 3.8 For each $\alpha \in J$ there exists a basis $\mathcal{S}_{\alpha}$ of $H_{1}^{\text {odd }}(\tilde{X}, \mathbb{Z})$ consisting of lifts of edges of the Delaunay triangulation such that the map $\Phi_{\alpha}=\Phi_{\mathcal{S}_{\alpha}}$ is defined on all of $W_{\alpha}^{\prime}$ and is a coordinate system on the part of $W_{\alpha}^{\prime}$ not contained in the multiple zero locus. 
Proof. See [MasSm91, §4].

We can now define a "Euclidean norm" on the tangent space of $\mathcal{Q}^{1} \mathcal{T}_{g}$ as follows: if $v$ is a tangent vector based at the point $q \in W_{\alpha}$ we define

$$
\|v\|_{E}=\left|\Phi_{\alpha}^{*}(v)\right|
$$

where $|\cdot|$ is the standard norm on $\mathbb{C}^{h}$. The norm $\|\cdot\|_{E}$ depends on the choices of the $\mathcal{S}_{\alpha}$ and the $W_{\alpha}^{\prime}$; however if $\|\cdot\|_{E}^{\prime}$ is the Euclidean norm obtained from a different choices, then the ratio of $\|\cdot\|_{E}$ and $\|\cdot\|_{E}^{\prime}$ is bounded by a constant depending only on $\mathcal{K}$.

The Euclidean Distance. We define the Euclidean length of a path $\gamma:[0, r] \rightarrow \mathcal{Q}^{1} \mathcal{T}_{g}$ to be $\int_{0}^{r}\left\|\gamma^{\prime}(t)\right\|_{E} d t$. We define the Euclidean distance $d_{E}\left(q, q^{\prime}\right)$ to be the infimum of the Euclidean length of paths connecting $q$ and $q^{\prime}$. Locally, up to a multiplicative constant, $d_{E}\left(q, q^{\prime}\right)=\left|\Phi_{\alpha}(q)-\Phi_{\alpha}\left(q^{\prime}\right)\right|$,

We denote the Teichmüller distance on $\mathcal{T}_{g}$ by $d_{\mathcal{T}}$. For $p \in \mathcal{Q}^{1} \mathcal{T}_{g}$ and $\epsilon>0$ let $B_{E}(p, \epsilon)$ denote the ball in the Euclidean metric of radius $\epsilon$ centered at $p$, and let $B_{\mathcal{T}}(\pi(p), \epsilon)$ denote the analogous ball in the Teichmüller metric. We note the following:

Lemma 3.9 There exist continuous functions $f_{i}: \mathbb{R}^{+} \rightarrow \mathbb{R}^{+}$with $f_{i}(r) \rightarrow 0$ as $r \rightarrow 0$ such that for any two points $p_{1}$ and $p_{2}$ in $\pi^{-1}(\mathcal{K})$ on the same leaf of $\mathcal{F}^{u u}$, we have

$$
f_{1}\left(d_{E}\left(p_{1}, p_{2}\right)\right) \leq d_{\mathcal{T}}\left(\pi\left(p_{1}\right), \pi\left(p_{2}\right)\right) \leq f_{2}\left(d_{E}\left(p_{1}, p_{2}\right)\right) .
$$

Proof. This follows from the compactness of $\mathcal{K}$.

Remark. The Euclidean distance has a number of useful properties: it behaves well near the multiple zero locus, and on compact subsets of $\mathcal{T}_{g}$ it is absolutely continuous with respect to the Teichmüller distance (see Lemma 3.9). However, often we need to deal instead with the modified Hodge distance because of its non-expansion and decay properties under the geodesic flow (Theorem 3.15 below).

The following theorem is the main result of this subsection:

Theorem 3.10 There exists constants $0<c_{1}<c_{2}$, both depending only on $\mathcal{K}$, such that for any $q_{1}, q_{2} \in \pi^{-1}(\mathcal{K})$ on the same leaf of $\mathcal{F}^{u u}$ with $d_{E}\left(q_{1}, q_{2}\right)<1$ we have:

$$
c_{1} d_{E}\left(q_{1}, q_{2}\right) \leq d_{H}\left(q_{1}, q_{2}\right) \leq c_{2} d_{E}\left(q_{1}, q_{2}\right)\left|\log d_{E}\left(q_{1}, q_{2}\right)\right|^{1 / 2} .
$$

Thus, in particular, on compact sets, the modified Hodge metric is equivalent to the Euclidean metric, and then, in view of Lemma 3.9. the modified Hodge metric on the restriction of a leaf of $\mathcal{F}^{u u}$ to $\pi^{-1}(\mathcal{K})$ is equivalent to the Teichmüller metric.

Note that the lower bound in Theorem 3.10 is clear since up to a constant depending only on $\mathcal{K}$, the modified Hodge norm is always bigger than the Euclidean norm, see (3.11) and (3.12). The rest of this subsection will consist of the proof of the upper bound in Theorem 3.10 For $q \in \mathcal{Q}^{1} \mathcal{T}_{g}$, let $\ell_{\min }(q)$ denote the length of the shortest saddle connection in the flat metric defined by $q$.

We will use the following standard fact about Delaunay triangulations:

Lemma 3.11 Suppose $T$ is the Delaunay triangulation of a surface in $q \in \mathcal{Q}^{1} \mathcal{T}_{g}$. Let $\ell_{\text {min }}(q)$ denote the length of the shortest saddle connection in $q$. Then any saddle connection in $q$ of length at most $\sqrt{2} \ell_{\text {min }}(q)$ is an edge of $T$. 
Proof. Let $z_{1}, \ldots, z_{n}$ denote the zeroes of $q$ (i.e. the conical points in the flat metric). For each $z_{i}$, let the Voronoi polygon $V_{i}$ denote the set of points of $q$ that are closer to $z_{i}$ then to any other $z_{j}$, $j \neq i$.

Recall that the Delaunay triangulation $T$ of $q$ is dual to the Voronoi diagram in the sense that $z_{i}$ and $z_{j}$ are connected by an edge of $T$ if $V_{i}$ and $V_{j}$ share a common edge (and also under some conditions if $V_{i}$ and $V_{j}$ share a common vertex).

Let $e$ be a saddle connection in $q$, connecting $z_{i}$ and $z_{j}$. (See Figure 11). Let $m$ be the midpoint of $e$. Let $k$ be such that $d\left(z_{k}, m\right)$ is minimal. Suppose $e$ is not an edge of the Delaunay triangulation $T$. Then $k \neq i, j$ and $d\left(m, z_{k}\right) \leq d\left(m, z_{i}\right)=\frac{1}{2} d\left(z_{i}, z_{j}\right)$. We may assume that the angle at $m$ between the segments $\overline{m z_{i}}$ and $\overline{m z_{k}}$ is smaller then $\pi / 2$ (otherwise replace $z_{i}$ by $z_{j}$ ). Let $p$ be the point on the segment $\overline{m z_{i}}$ such that $d(p, m)=d\left(p, z_{k}\right)$. Now consider the isosceles acute triangle $\tau$ whose vertices are $m, p$ and $z_{k}$. This triangle cannot contain any zeroes (or else $z_{k}$ would not be the saddle connection closest to $m)$. Therefore, $d\left(p, z_{k}\right) \leq \sqrt{2} d\left(m, z_{k}\right)$. Hence,

$$
\begin{aligned}
\ell_{\text {min }}(q) \leq d\left(z_{i}, z_{k}\right) & \leq d\left(z_{i}, p\right)+d\left(p, z_{k}\right) \leq\left(d\left(z_{i}, m\right)-d\left(m, z_{k}\right)\right)+\sqrt{2} d\left(m, z_{k}\right) \\
= & \frac{1}{2} d\left(z_{i}, z_{j}\right)+(\sqrt{2}-1) d\left(m, z_{k}\right) \leq \frac{1}{2} d\left(z_{i}, z_{j}\right)+\frac{(\sqrt{2}-1)}{2} d\left(z_{i}, z_{j}\right)=\frac{\sqrt{2}}{2} d\left(z_{i}, z_{j}\right) .
\end{aligned}
$$

Thus, if $e$ is not an edge of the Delaunay triangulation, then $\ell(e) \geq \sqrt{2} \ell_{\min }(q)$.

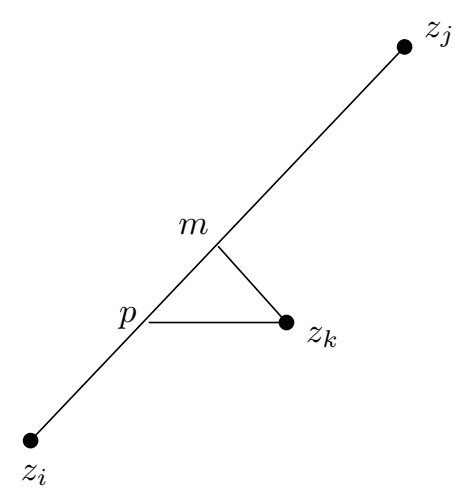

Figure 1. Proof of Lemma 3.11.

Recall that an integral multicurve is a finite set of oriented simple closed curves with integral weights. By convention, a negative weight corresponds to reversing the orientation.

Lemma 3.12 Suppose $T$ is a geodesic triangulation of an orientable surface $\omega$ in $\Omega^{1} \mathcal{T}_{g}$. We orient each edge of $T$ so that its $x$ component is positive (for a vertical edge the orientation is not defined). Suppose $W$ is a subset of the edges of $T$. Then there exists an integral multicurve $\Delta$ on $\omega$ such that

(a) $\Delta$ is disjoint from the vertices of $T$ and is transverse to the edges.

(b) $\Delta$ crosses each edge of $W$ at least once. 
(c) Each time $\Delta$ crosses an edge of $e \in W$, the crossing is from left to right (with respect to the orientation on the edge). If e is vertical, then all crossings must be from the same side.

(d) For each edge $e$ of $T$, the intersection number $i(\Delta, e) \leq n$, where $n$ depends only on the genus $g$.

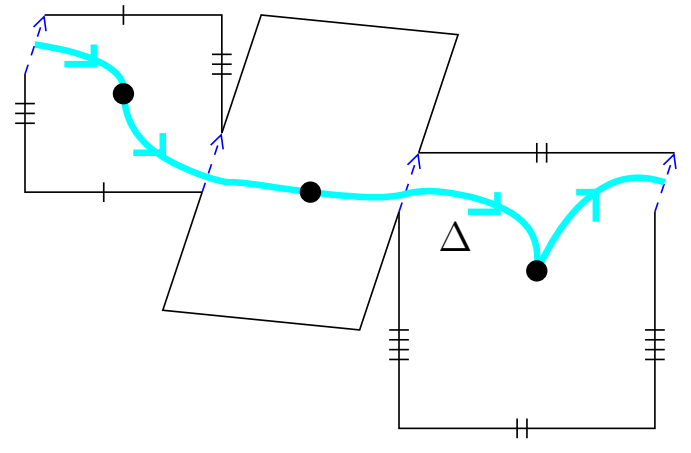

Figure 2. Lemma 3.12 The surface consists of three flat tori glued to each other as shown. The set $W$ consists of the three dotted lines separating the tori. (The two dotted lines on the opposite sides of the figure are identified). The multicurve (in this case closed curve) $\Delta$ is drawn. The black dots are vertices of the graph $\mathcal{G}$ (used in the proof).

Proof. Each triangulated surface which has vertical edges is the limit of triangulated surfaces which do not. Hence, without loss of generality, we may assume that $T$ has no vertical edges.

We define a directed graph $\mathcal{G}$ as follows (See Figure 2). The vertices of $\mathcal{G}$ are the connected components of $\omega \backslash W$. Two vertices $A$ and $B$ of $\mathcal{G}$ are connected by an directed edge of $\mathcal{G}$ if and only if there exists a saddle connection $\gamma \in W$ such that $A$ is incident to $\gamma$ from the left and $B$ is incident to $\gamma$ from the right.

We now claim that for each pair $A, B$ of vertices of $\mathcal{G}$ there exists at least one directed path from $A$ to $B$. This can be derived from the minimality of the flow in an almost vertical direction, but we prefer to give a direct combinatorial argument. Suppose this is not true. Let $\mathcal{A}$ be the set of vertices of $\mathcal{G}$ which can be reached from $A$ by a directed path. Then $A \in \mathcal{A}$ and $B \in \mathcal{A}^{c}$; in particular $\mathcal{A}$ and $\mathcal{A}^{c}$ are both non-empty. Now let $D$ be the closure of the connected components in $\omega \backslash W$ corresponding to vertices of $\mathcal{A}$. Then $D$ is a subsurface of $\omega$, and $\partial D$ consists of edges from $W$. For each edge $\gamma \in \partial D$ let $\epsilon_{\gamma}=+1$ if $D$ is on the left of $\gamma$ and -1 otherwise. Then, (since $D$ is orientable) $\sum_{\gamma \in \partial D} \epsilon_{\gamma} \gamma=0$. It follows that there exists $\gamma \in \partial D$ such that the $x$ component of $\epsilon_{\gamma} \gamma$ is positive. Since the $x$ component of $\gamma$ is positive, this implies that $\epsilon_{\gamma}=1$. Thus the directed edge of $\mathcal{G}$ corresponding to $\gamma$ is directed from $D$ to $D^{c}$. This means that some vertex of $\mathcal{G}$ outside of $\mathcal{A}$ can be reached from a vertex in $\mathcal{A}$ by a directed path, contradicting the definition of $\mathcal{A}$.

Thus, every pair of vertices of $\mathcal{G}$ can be connected by a directed path. This implies every directed edge of $\mathcal{G}$ is contained in a directed cycle of $\mathcal{G}$. Therefore there exists a finite union $\Delta$ of directed cycles such that every edge in $\mathcal{G}$ is contained in $\Delta$. The length of $\Delta$ is bounded in terms of the size of $\mathcal{G}$, i.e. in terms of the genus. We can thus realize $\Delta$ as a multicurve on $\omega$ with properties (a)-(d). 
Lemma 3.13 There exist $\rho_{1}>0$ and $C>0$ depending only on $\mathcal{K}$ such that for any $q \in \pi^{-1}(\mathcal{K})$ there exists $q^{\prime} \in \pi^{-1}(\mathcal{K}) \cap \mathcal{F}^{u u}(q)$ and a path $\gamma:\left[0, \rho_{1}\right] \rightarrow \mathcal{F}^{u u}(q)$ such that $\gamma(0)=q, \gamma\left(\rho_{1}\right)=q^{\prime}$, and for $t \in\left[0, \rho_{1}\right]$,

$$
\ell_{\min }(\gamma(t)) \geq \ell_{\min }(\gamma(0)) \sqrt{1+t^{2}}
$$

and also

$$
d_{H}\left(q, q^{\prime}\right) \leq C \int_{0}^{\rho_{1}}\left|\log \left(\ell_{\min }(q) \sqrt{1+t^{2}}\right)\right|^{1 / 2} d t
$$

Proof. Let $\tilde{S}=(\tilde{X}, \tilde{\omega})$ denote the double cover corresponding to $q$. Consider the Delaunay triangulation of $\tilde{S}$. By Lemma 3.11 all saddle connections of length at most $\sqrt{2} \ell_{\text {min }}(\tilde{S})$ belong to the Delaunay triangulation. Let $W$ denote this set of saddle connections. Let $\Delta$ be the multicurve obtained by applying Lemma 3.12 to $\tilde{S}$ and $W$.

Let $\tau$ be the involution corresponding to $q$. Let $\Delta^{\prime}=\Delta-\tau(\Delta)$. Then $\Delta^{\prime}$ also has properties (a)-(d) of Lemma 3.12 In addition, $\tau\left(\Delta^{\prime}\right)=-\Delta^{\prime}$.

Let $\rho_{1} \in(0, \sqrt{2})$ be a constant which will be chosen later (depending only on the genus). We now use $\Delta^{\prime}$ to define a path $\gamma:\left[0, \rho_{1}\right] \rightarrow \mathcal{Q}^{1} \mathcal{T}_{g}$ contained in $\pi^{-1}(\mathcal{K})$ and staying on the same leaf of $\mathcal{F}^{u u}$. We will actually define the path $\tilde{\gamma}(t)$ where for each $t, \tilde{\gamma}(t)$ is the double cover of $\gamma(t)$. Let $\tilde{S}$ denote the double cover of $q$, and set $\tilde{\gamma}(0)=\tilde{S}$. For each $t \in\left[0, \rho_{1}\right], \tilde{\gamma}(t)$ is built from the same triangles as $\tilde{S}$, but for each edge $e$, we add $I\left(e, \Delta^{\prime}\right) t \ell_{\min }(q)$ to the $x$-component, where $I(\cdot, \cdot)$ denotes the algebraic intersection number (cf. MasSm91, §6]).

Note that we do not assert that the Delaunay triangulation of $\tilde{\gamma}(t)$ is the same as that of $\tilde{S}=\tilde{\gamma}(0)$. However, because of the form of $\Delta^{\prime}$, the involution $\tau$ acts on $\tilde{\gamma}(t)$, and we can let $\gamma(t)$ be the quotient by $\tau$.

We now claim that (3.18) holds. Indeed, if $e \in W$ is a saddle connection in $\tilde{\gamma}(0)$ with vector $\left(x_{0}, y_{0}\right)$, then $x_{0} \geq 0$, and on $\tilde{\gamma}(t), e$ has length

$$
\sqrt{y_{0}^{2}+\left(x_{0}+I\left(e, \Delta^{\prime}\right) \ell_{\min }(q) t\right)^{2}} \geq \sqrt{\ell_{\min }(q)^{2}+\ell_{\min }(q)^{2} t^{2}}=\ell_{\min }(q) \sqrt{1+t^{2}} .
$$

Suppose $t_{1}, t_{2} \in\left[0, \rho_{1}\right]$ and $\eta$ are such that $\eta$ is a saddle connection on $\tilde{\gamma}(t)$ for $t_{1}<t<t_{2}$. Let $\ell_{t}(\eta)$ denote the length of $\eta$ in the flat metric on $\tilde{\gamma}(t)$. Then,

$$
\ell_{t}(\eta) \geq \ell_{t_{1}}(\eta)-|I(\eta, \Delta)| \ell_{\min }(q)\left(t-t_{1}\right) .
$$

Now suppose $z$ and $w$ be any two zeroes of $q$. Let $\lambda_{t}(z, w)$ denote the shortest path between $z$ and $w$ on $\tilde{\gamma}(t)$, and let $\left|\lambda_{t}(z, w)\right|$ denote the length of $\lambda_{t}(z, w)$, i.e. the distance between $z$ and $w$ in the flat metric on $\tilde{\gamma}(t)$. Suppose $\lambda_{0}(z, w)$ is not an edge in $W$. Then, either $\lambda_{0}(z, w)$ is a saddle connection not in $W$, in which case $\left|\lambda_{0}(z, w)\right| \geq \sqrt{2} \ell_{\min }(q)$, or $\lambda_{0}(z, w)$ is a union of at least two saddle connections, so that $\left|\lambda_{0}(z, w)\right| \geq 2 \ell_{\min }(q) \geq \sqrt{2} \ell_{\min }(q)$. It now follows from (3.21) that for all $t \in\left[0, \rho_{1}\right]$,

$$
\left|\lambda_{t}(z, w)\right| \geq(\sqrt{2}-n m t) \ell_{\min }(q),
$$

where $n$ is the maximum intersection number of $\Delta^{\prime}$ with a saddle connection in the Delaunay triangulation of $\tilde{S}$, and $m$ is the maximal number of saddle connections in $\lambda_{t}(z, w)$. Note that both $n$ and $m$ are bounded by the genus. Now we choose $\rho_{1}$ so that $\sqrt{2}-n m \rho_{1} \geq\left(1+\rho_{1}\right)^{2}$. Then, if $\lambda_{0}(z, w)$ is not a saddle connection in $W$, then for all $t \in\left[0, \rho_{1}\right]$,

$$
\left|\lambda_{t}(z, w)\right| \geq \ell_{\min }(\gamma(0)) \sqrt{1+t^{2}} .
$$


Now (3.18) follows from (3.20) and (3.22).

We now estimate the Hodge length of the path $\gamma:\left[0, \rho_{1}\right] \rightarrow \mathcal{Q}^{1} \mathcal{T}_{g}$. By Lemma 3.4 and Lemma 3.5.

$$
\ell_{\gamma(t)}(\sigma) \geq \frac{C_{g}}{\left|\log \left(\ell_{\min }(q) \sqrt{1+t^{2}}\right)\right|}
$$

where $\ell_{\sigma}(S)$ is defined to be the infimum over all simple closed curves $\alpha$ of $\ell_{\alpha}(\sigma)$ (and $\sigma$ is the hyperbolic metric in the conformal class of $S$ ). By construction, the intersection number of $\Delta^{\prime}$ with a short basis (see 33.3.2) of $\gamma(t)$ is bounded depending only on the genus. Therefore, by (3.9) and (3.10),

$$
\left\|\gamma^{\prime}(t)\right\|^{\prime} \leq C\left|\log \left(\ell_{\min }(q) \sqrt{1+t^{2}}\right)\right|^{1 / 2},
$$

where $C$ depends only on the genus. Thus, if $q_{1}=\gamma\left(\rho_{1}\right)$, then

$$
d_{H}\left(q, q_{1}\right) \leq \int_{0}^{\rho_{1}}\left\|\gamma^{\prime}(t)\right\|^{\prime} d t \leq \int_{0}^{\rho_{1}} C\left|\log \left(\ell_{\min }(q) \sqrt{1+t^{2}}\right)\right|^{1 / 2} d t .
$$

Thus (3.19) holds.

Lemma 3.14 There exists $\epsilon_{0}>0$ (depending on $\mathcal{K}$ ) such that for all $\epsilon<\epsilon_{0}$ and for all $q \in \pi^{-1}(\mathcal{K})$ with $\ell_{\min }(q)<\epsilon$, there exists $q^{\prime}$ on the same leaf of $\mathcal{F}^{u u}$ as $q$ with $\ell_{\min }\left(q^{\prime}\right) \geq \epsilon$, and $d_{H}\left(q, q^{\prime}\right) \leq$ $k \epsilon|\log \epsilon|^{1 / 2}$, where $k$ depends only on $\mathcal{K}$.

Proof. We define a sequence $q_{n}$ as follows: let $q_{0}=q$. If $q_{n}$ has been defined already, we apply Lemma 3.13 with $q=q_{n}$, and define $q_{n+1}$ to be the point $q^{\prime}$ guaranteed by Lemma 3.13 We obtain a sequence $q_{n}$ with

$$
\ell_{\min }\left(q_{n+1}\right) \geq \ell_{\min }\left(q_{n}\right)\left(1+\rho_{1}^{2}\right)^{1 / 2}=\rho_{2} \ell_{\min }\left(q_{n}\right),
$$

where we let $\rho_{2}=\left(1+\rho_{1}^{2}\right)^{1 / 2}$. Define $t_{n}$ inductively by $t_{0}=0, t_{n+1}=t_{n}+\rho_{1} \ell_{\min }\left(q_{n}\right), n \geq 0$. Then,

$$
t_{n}=\rho_{1} \sum_{k=0}^{n-1} \ell_{\min }\left(q_{k}\right) \leq \rho_{1} \sum_{k=0}^{n-1} \frac{\ell_{\min }\left(q_{n}\right)}{\rho_{2}^{n-k}}=\rho_{1} \ell_{\min }\left(q_{n}\right) \frac{\rho_{2}^{-1}-\rho_{2}^{-n-1}}{1-\rho_{2}^{-1}} \leq \frac{\rho_{1} \ell_{\min }\left(q_{n}\right)}{\rho_{2}-1} .
$$

Then,

$$
t_{n+1}=t_{n}+\rho_{1} \ell_{\min }\left(q_{n}\right) \leq \frac{\rho_{1} \rho_{2}}{\rho_{2}-1} \ell_{\min }\left(q_{n}\right) .
$$

Thus, for $t \in\left[t_{n}, t_{n+1}\right]$,

$$
\ell_{\min }\left(q_{n}\right) \geq \frac{\rho_{2}-1}{\rho_{1} \rho_{2}} t \equiv \rho_{3} t
$$

We have

$$
d_{H}\left(q_{n}, q_{n+1}\right) \leq \int_{t_{n}}^{t_{n+1}} C\left|\log \left(\ell_{\min }\left(q_{n}\right) \sqrt{1+t^{2}}\right)\right|^{1 / 2} d t \leq \int_{t_{n}}^{t_{n+1}} C\left|\log \left(\rho_{3} t \sqrt{1+t^{2}}\right)\right|^{1 / 2} d t .
$$

Thus,

$$
d_{H}\left(q_{0}, q_{n}\right) \leq \int_{0}^{t_{n}} C\left|\log \left(\rho_{3} t \sqrt{1+t^{2}}\right)\right|^{1 / 2} d t
$$


We choose $n$ so that $\ell_{\min }\left(q_{n}\right)$ is comparable to $\epsilon$. Let $q^{\prime}=\gamma\left(t_{n}\right)$. Then, $\ell_{\min }\left(q^{\prime}\right) \geq t_{n} \approx \epsilon$. Now the modified Hodge length of the path $q_{0}, q_{1}, \ldots, q_{n}$ is $O\left(\epsilon|\log \epsilon|^{1 / 2}\right)$.

Proof of Theorem 3.10. Since $\mathcal{K}$ is compact, the intersection of the multiple zero locus with $\pi^{-1}(\mathcal{K})$ is (contained in) finite union of hyperplanes $H_{1}, \ldots, H_{n}$. Each hyperplane $H_{j}$ has complex codimension 1 . We can choose $\epsilon_{0}>0$ such that any two $H_{j}$ which do not intersect in $\pi^{-1}(\mathcal{K})$ are at least $\epsilon_{0}$ apart. Let $\delta_{0}>0$ be a lower bound on the angle between any two $H_{j}$ which do intersect in $\pi^{-1}(\mathcal{K})$. Clearly $\epsilon_{0}$ and $\delta_{0}$ depend only on $\mathcal{K}$.

Let $Z$ be the locus where our quadratic differential has either a zero of order at least 3 or two zeroes each of order at least 2 . Then there exists a constant $k_{0}$ (depending only on $\delta_{0}$ and thus only on $\mathcal{K}$ ) such that for any $q \in \pi^{-1}(\mathcal{K})$ and any $\epsilon>0$, if the ball $B_{E}(q, \epsilon)$ intersects at least two hyperplanes $H_{j}$ then $d_{E}(q, Z)<k_{0} \epsilon$.

Take two points $q_{1}, q_{2} \in \pi^{-1}(\mathcal{K})$ on the same leaf of $\mathcal{F}^{u u}$, with $d\left(q_{1}, q_{2}\right)=\epsilon$. Choose $k_{3}>k_{0}$ (thus $k_{3}$ depends only on $\mathcal{K}$ ). We also assume that $k_{3} \epsilon<\epsilon_{0}$. By Lemma 3.14 (with $k_{3} \epsilon$ in place of $\epsilon$ ), there exist $q_{1}^{\prime}, q_{2}^{\prime}$ so that for $i=1,2$, we have $d_{H}\left(q_{i}, q_{i}^{\prime}\right) \leq k_{1} \epsilon|\log \epsilon|^{1 / 2}, d_{E}\left(q_{i}, q_{i}^{\prime}\right) \leq k_{2} \epsilon$, and $\ell_{\text {min }}\left(q_{i}\right)=k_{3} \epsilon$, where $k_{1}, k_{2}$ depend only on $\mathcal{K}$. Since $k_{3}>k_{0}$ and $k_{3} \epsilon<\epsilon_{0}$, there exist $\epsilon_{1}, \epsilon_{1}^{\prime}$ such that $k_{3} \epsilon / 2>\epsilon_{1}>\epsilon_{1}^{\prime}>\epsilon /\left(2 k_{3}\right)$, and for all $q \in \pi^{-1}(\mathcal{K})$, either $d_{E}(q, Z)<\epsilon_{1}$ or $B_{E}\left(q, \epsilon_{1}^{\prime}\right)$ contains at most one hyperplane from the multiple zero locus.

Note that the intersection of $Z$ with $\pi^{-1}(\mathcal{K})$ has complex codimension at least 2 . Hence, the intersection of the $\epsilon_{1}$-neighborhood of $Z$ with $\mathcal{F}^{u u}\left(q_{1}\right) \cap \pi^{-1}(\mathcal{K})$ is contained in the $\epsilon_{1}$-neighborhood of a finite union of hyperplanes, each of real codimension at least 2 . Then there exists a constant $k_{4}$ depending only on $\mathcal{K}$, and a path $\gamma$ connecting $q_{1}^{\prime}$ to $q_{2}^{\prime}$ of length at most $k_{4} \epsilon$, which avoids the $\epsilon_{1}$-neighborhood of $Z$.

Now let $p_{0}=q_{1}^{\prime}$, and mark points $p_{i}$ along $\gamma$ which are $\epsilon_{1}^{\prime} / 2$ apart in the Euclidean metric. We have $p_{n}=q_{2}^{\prime}$. Let $B_{i}$ be the ball of Euclidean diameter $\epsilon_{1}^{\prime} / 2$ which contains $p_{i}$ and $p_{i+1}$ on its boundary. By construction $B_{i}$ contains at most one hyperplane (which we will denote $L$ ) from the multiple zero locus. Note that by Lemma 3.4 Lemma 3.5 and (3.12), for any $p \in B_{i}$ and any tangent vector $v$ at $p$, the modified Hodge norm of $v$ can be estimated as

$$
\|v\|_{H}^{\prime} \leq C\left|\log d_{E}(p, L)\right|^{1 / 2}\|v\|_{E},
$$

where $\|v\|_{E}$ is the Euclidean norm of $v$, and $d(p, L)$ denotes the Euclidean distance between the point $p$ and the hyperplane $L$.

Let $p_{i}^{\prime}$ be the farthest point in $B_{i}$ from the hyperplane. Then, after connecting $p_{i}$ and $p_{i}^{\prime}$ by a straight line path and using (3.23), we see that $d_{H}\left(p_{i}, p_{i}^{\prime}\right)=O\left(\epsilon|\log \epsilon|^{1 / 2}\right)$, and also $d_{H}\left(p_{i}^{\prime}, p_{i+1}\right)=$ $O\left(\epsilon|\log \epsilon|^{1 / 2}\right)$. Thus, since the number of $B_{i}$ along the path is bounded by a constant depending only on $\mathcal{K}$, we finally obtain

$$
d_{H}\left(q_{1}^{\prime}, q_{2}^{\prime}\right)=O\left(d_{E}\left(q_{1}^{\prime}, q_{2}^{\prime}\right)|\log \epsilon|^{1 / 2}\right)
$$

\subsection{The Non-expansion and Decay of the Hodge Distance.}

Theorem 3.15 Suppose $q \in \mathcal{Q}^{1} \mathcal{T}_{g}$ and $q^{\prime} \in \mathcal{Q}^{1} \mathcal{T}_{g}$ are in the same leaf of $\mathcal{F}^{s s}$. Then 
(a) There exists a constant $c_{H}>0$ such that for all $t \geq 0$,

$$
d_{H}\left(g_{t} q, g_{t} q^{\prime}\right) \leq c_{H} d_{H}\left(q, q^{\prime}\right) .
$$

(b) Suppose $\epsilon>2 \epsilon_{1}$, where $\epsilon_{1}$ is as in the definition of short basis (see 3 3.3.2). Let $K=\{q \epsilon$ $\left.\mathcal{Q}^{1} \mathcal{T}_{g}: \ell_{\min }(q)>\epsilon\right\}$. Suppose $d_{H}\left(q, q^{\prime}\right)<1$, and $t>0$ is such that

$$
\left|\left\{s \in[0, t]: g_{s} q \in \Gamma K\right\}\right| \geq(1 / 2) t .
$$

Then for all $0<s<t$,

$$
d_{H}\left(g_{s} q, g_{s} q^{\prime}\right) \leq C e^{-c s} d_{H}\left(q, q^{\prime}\right),
$$

where $c$ and $C$ depend only on $g$ and $\epsilon$.

Proof. The statement (a) follows immediately from Theorem 3.6. For the second statement, let $\gamma:[0, \rho] \rightarrow \mathcal{Q}^{1} \mathcal{T}_{g}$ be a modified Hodge length minimizing path connecting $q$ to $q^{\prime}$ (and staying in the same leaf of $\left.\mathcal{F}^{s s}\right)$. We assume that $\gamma$ is parametrized so that $d_{H}(\gamma(u), q)=u$. By assumption, $\rho<1$.

Let $N=4 c_{H} /\left(c_{1} \epsilon \rho\right)$, where $c_{1}$ is as in Theorem 3.10. For $0 \leq j \leq N$, let $u_{j}=j c_{1} \epsilon /\left(4 c_{H}\right)$. We now claim that for all $j$ there exists $t_{j}$ such that for $s>t_{j}$ such that $g_{s} q \in \Gamma K$, we have for all $0 \leq u<u_{j}$,

$$
d_{E}\left(g_{s} \gamma(u), g_{s} q\right)<\epsilon \sum_{k=0}^{j} 2^{-k},
$$

and

$$
d_{H}\left(g_{s} \gamma(u), g_{s} q\right)<C e^{-c s} u,
$$

where $C$ and $c$ depend only on $g$ and $\epsilon$. The equations (3.25) and (3.26) will be proved by induction on $j$. If $j=0$ then there is nothing to prove. Now assume $j \geq 1$, and (3.25) and (3.26) are true for all $u \leq u_{j-1}$. Suppose $u<u_{j}$. By (a), we have for all $t>0$,

$$
d_{H}\left(g_{t} \gamma(u), g_{t} \gamma\left(u_{j-1}\right)\right)<c_{H}\left(u-u_{j-1}\right)<c_{1} \epsilon / 4 .
$$

Therefore by Theorem 3.10 $d_{E}\left(g_{t} \gamma(u), g_{t} \gamma\left(u_{j-1}\right)\right)<\epsilon / 2$. Therefore, by the inductive assumption (3.25), for the $s>t_{j-1}$ such that $g_{s} q \in \Gamma K$, for all $u_{j-1}<u<u_{j}$, we have $\ell_{\min }\left(g_{s} \gamma(u)\right)>\epsilon / 2$. Thus, by (3.14), for all $u_{j-1}<u<u_{j}$, the modified Hodge norm of $\gamma^{\prime}(u)$ is within a constant of the Hodge norm of $\gamma^{\prime}(u)$. Then by Theorem 3.3, for all $u_{j-1}<u<u_{j}$,

$$
d_{H}\left(g_{t} \gamma(u), g_{t} \gamma\left(u_{j-1}\right)\right)<C e^{-c t} d_{H}\left(\gamma(u), \gamma\left(u_{j-1}\right)\right) .
$$

Therefore, by Theorem 3.10, there exists $t_{j}>0$ (depending only on $g$ and $\epsilon$ ) such that for $s>t_{j}$ with $g_{s} q \in \Gamma K$, and $u_{j-1} \leq u<u_{j}$, (3.25) holds. Also (3.26) follows from (3.27). The induction terminates after finitely many steps (depending only on $g$ and $\epsilon$ ). Thus (b) holds. 


\section{The multiple zero locus.}

In this section we prove Theorem 2.6 and Theorem 2.7

Let $\mathcal{K}$ be a compact subset of $\mathcal{T}_{g}$. We assume that $\mathcal{K}$ is contained in one fundamental domain for the action of $\Gamma$ on $\mathcal{T}_{g}$ (and thus we can identify $\mathcal{K}$ with a subset of $\mathcal{M}_{g}$ ). All of our implied constants will depend on $\mathcal{K}$.

Notation. Suppose $W \subset \mathcal{Q}^{1} \mathcal{T}_{g}$ and $s>0$. Let $W(s)$ denote the set of $q \in \mathcal{Q}^{1} \mathcal{T}_{g}$ such that there exists $q^{\prime} \in W$ on the same leaf of $\mathcal{F}^{u u}$ as $q$ such that $d_{H}\left(q, q^{\prime}\right)<s$. Recall that for a subset $A \subset \mathcal{T}_{g}$, $\operatorname{Nbhd}_{r}(A)$ denotes the set of points within Teichmüller distance $r$ of $A$. We will also use $A(r)$ to denote $\operatorname{Nbhd}_{r}(A)$. Let $\bar{\nu}$ and $\eta^{+}$be as in $\$ 2.2$, so that so that for a set $F$ contained in a leaf of $\mathcal{F}^{u u}$, $\bar{\nu}\left(\eta^{+}(F)\right)=\mu_{\alpha^{u u}}(F)$.

Lemma 4.1 Suppose $U \subset \pi^{-1}(\mathcal{K}), \delta>0$ and $t>0$. Let $W=g_{t} U \cap \Gamma \pi^{-1}(\mathcal{K})$. There is a $C(\delta)>0$ so that

$$
\mathbf{m}\left(N b h d_{2}(\pi(W))\right) \leq C(\delta) \bar{\nu}\left(\eta^{+}(W(\delta))\right),
$$

(By our convention, the constant $C(\delta)$ depends on $\mathcal{K}$ as well). Also

$$
\bar{\nu}\left(\eta^{+}(W(1))\right) \leq C(\delta) \bar{\nu}\left(\eta^{+}(W(\delta))\right) .
$$

Proof. Let $\delta_{0}=\delta_{0}(\mathcal{K}, \delta)$ be a constant to be chosen later. We decompose $U$ into pieces $U_{\alpha}$ such that each piece is within (modified) Hodge distance $\delta_{0} / 2$ of a single leaf of $\mathcal{F}^{u u}$. Let

$$
W_{\alpha}=g_{t} U_{\alpha} \cap \Gamma \pi^{-1}(\mathcal{K}) .
$$

Then

$$
\mathbf{m}\left(N b h d_{2}(\pi(W))\right) \leq \sum_{\alpha} \mathbf{m}\left(N b h d_{2}\left(\pi\left(W_{\alpha}\right)\right)\right)
$$

In view of Theorem 3.10 the number of pieces is bounded depending only on $\mathcal{K}$ and $\delta$ (since $\delta_{0}=$ $\delta_{0}(\mathcal{K}, \delta)$ ). Thus, it is enough to show that (4.1) holds with $W$ replaced by $W_{\alpha}$.

We now claim that without loss of generality, we may assume that $U_{\alpha}$ has the following "product property": given $q_{1}, q_{2} \in U_{\alpha}$, there exists $q_{2}^{\prime} \in U_{\alpha}$ on the same leaf of $\mathcal{F}^{u u}$ as $q_{1}$ and on the same leaf of $\mathcal{F}^{s}$ as $q_{2}$. If not, let

$$
U_{\alpha}^{\prime}=U_{\alpha} \cup\left\{\mathcal{F}^{u u}\left(q_{1}\right) \cap \mathcal{F}^{s}\left(q_{2}\right): q_{1}, q_{2} \in U_{\alpha}\right\} .
$$

Then $\eta^{+}\left(U_{\alpha}\right)=\eta^{+}\left(U_{\alpha}^{\prime}\right)$, and therefore $\eta^{+}\left(g_{t} U_{\alpha}\right)=\eta^{+}\left(g_{t} U_{\alpha}^{\prime}\right)$. Also if $\delta^{\prime}=\delta^{\prime}(\delta, K)$ is sufficiently small then $W_{\alpha}^{\prime}=g_{t} U_{\alpha}^{\prime} \cap \Gamma \pi^{-1}(\mathcal{K})$ satisfies $W_{\alpha}^{\prime}\left(\delta^{\prime}\right) \subset W_{\alpha}(\delta)$. Therefore we can proceed with the rest of the proof with $\delta^{\prime}$ instead of $\delta$ and $U_{\alpha}^{\prime}$ instead of $U_{\alpha}$. Therefore, without loss of generality, we may assume that $U_{\alpha}$ has the product property. Therefore, $g_{t} U_{\alpha}$ also has the product property.

Pick a maximal $\Delta \subset \pi\left(W_{\alpha}\right)$ such that for any two distinct $X, Y \in \Delta, d_{\mathcal{T}}(X, Y)=1$. Then,

$$
\operatorname{Nbhd}_{2}\left(\pi\left(W_{\alpha}\right)\right) \subset \bigcup_{X \in \Delta} B_{\tau}(X, 3),
$$

and hence,

$$
\mathbf{m}\left(N b h d_{2}\left(\pi\left(W_{\alpha}\right)\right)\right) \leq \mathbf{m}\left(\bigcup_{X \in \Delta} B_{\tau}(X, 3)\right) \leq \sum_{X \in \Delta} \mathbf{m}(B(X, 3)) \leq C(\mathcal{K})|\Delta|,
$$


where $|\Delta|$ denotes the cardinality of $\Delta$, and we have used the fact that $\Delta \subset \Gamma \mathcal{K}$.

For each $X \in \Delta$, pick one $q \in W_{\alpha} \cap \pi^{-1}(X)$. Let $\Delta^{\prime} \subset W_{\alpha}$ denote the resulting set of $q$ 's. Let $B_{E}^{u u}(q, r)$ denote the set of $q^{\prime} \in \mathcal{Q}^{1} \mathcal{T}_{g}$ on the same leaf of $\mathcal{F}^{u u}$ as $q$ with $d_{E}\left(q, q^{\prime}\right)<r$. We claim that for $\delta_{0}$ sufficiently small, we can pick $\delta_{2}$ depending only on $\mathcal{K}$ such that for all distinct pairs $q_{1}, q_{2} \in \Delta^{\prime}$,

$$
\eta^{+}\left(B_{E}^{u u}\left(q_{1}, \delta_{2}\right)\right) \cap \eta^{+}\left(B_{E}^{u u}\left(q_{2}, \delta_{2}\right)\right)=\emptyset .
$$

To prove (4.4), suppose $q_{1}, q_{2} \in \Delta^{\prime}$, and $q_{1} \neq q_{2}$. Let $q_{2}^{\prime}$ be such that $q_{1}$ and $q_{2}^{\prime}$ are on the same leaf of $\mathcal{F}^{u u}$ and $q_{2}$ and $q_{2}^{\prime}$ are on the same leaf of $\mathcal{F}^{s}$. By Theorem 3.15, we have $d_{H}\left(q_{2}, q_{2}^{\prime}\right)<c_{H} \delta_{0}$. Therefore, by Theorem 3.10 and Lemma 3.9. we can choose $\delta_{0}$ small enough so that $d_{\mathcal{T}}\left(\pi\left(q_{2}\right), \pi\left(q_{2}^{\prime}\right)\right) \leq 1 / 5$. Hence, $\pi\left(q_{2}^{\prime}\right) \subset \Gamma \mathcal{K}^{\prime}$ where $\mathcal{K}^{\prime} \subset \mathcal{T}_{g}$ is compact, and by the triangle inequality,

$$
d_{\mathcal{T}}\left(\pi\left(q_{1}\right), \pi\left(q_{2}^{\prime}\right)\right) \geq d_{\mathcal{T}}\left(\pi\left(q_{1}\right), \pi\left(q_{2}\right)\right)-d_{\mathcal{T}}\left(\pi\left(q_{2}\right), \pi\left(q_{2}^{\prime}\right)\right) \geq 4 / 5 .
$$

By Lemma 3.9 there exists $\delta_{1}>0$ (depending only on $\mathcal{K}^{\prime}$ ) such that for all $q, q^{\prime} \in \mathcal{K}^{\prime}$ on the same leaf of $\mathcal{F}^{u u}$ with $d_{E}\left(q, q^{\prime}\right)<\delta_{1}$, we have $d_{\mathcal{T}}\left(\pi(q), \pi\left(q^{\prime}\right)\right)<1 / 5$. Then, by (4.5) and the triangle inequality, $B_{E}^{u u}\left(q_{1}, \delta_{1}\right)$ and $B_{E}^{u u}\left(q_{2}^{\prime}, \delta_{1}\right)$ are disjoint. It follows that as subsets of $\mathcal{P} \mathcal{M F}$,

$$
\eta^{+}\left(B_{E}^{u u}\left(q_{1}, \delta_{1}\right)\right) \cap \eta^{+}\left(B_{E}^{u u}\left(q_{2}^{\prime}, \delta_{1}\right)\right)=\emptyset
$$

Let $\mathcal{F}^{s}(q)$ denote the leaf of $\mathcal{F}^{s}$ through $q$. Since $\eta^{+}$is continuous and $\mathcal{K}^{\prime}$ is compact, there exist constants $0<\delta_{2}<\delta_{1}$ and $\delta_{3}>0$ (depending only on $\mathcal{K}^{\prime}$ ) such that for all $q, q^{\prime} \in \mathcal{K}^{\prime}$ with $q \in \mathcal{F}^{s}\left(q^{\prime}\right)$ and $d_{H}\left(q, q^{\prime}\right)<\delta_{3}$, we have, as subsets of $\mathcal{P} \mathcal{M F}$,

$$
\eta^{+}\left(B_{E}^{u u}\left(q, \delta_{2}\right)\right) \subset \eta^{+}\left(B_{E}^{u u}\left(q^{\prime}, \delta_{1}\right)\right) .
$$

We now choose $\delta_{0}<\delta_{3} / c_{H}$. Then, since $q_{2}$ and $q_{2}^{\prime}$ are on the same leaf of $\mathcal{F}^{s}$ and $d_{H}\left(q_{2}, q_{2}^{\prime}\right)<$ $c_{H} \delta_{0}<\delta_{3}$, we have

$$
\eta^{+}\left(B_{E}^{u u}\left(q_{2}, \delta_{2}\right)\right) \subset \eta^{+}\left(B_{E}^{u u}\left(q_{2}^{\prime}, \delta_{1}\right)\right) .
$$

Now using (4.6), we get that as subsets of $\mathcal{P} \mathcal{M F}$,

$$
\eta^{+}\left(B_{E}^{u u}\left(q_{1}, \delta_{2}\right)\right) \cap \eta^{+}\left(B_{E}^{u u}\left(q_{2}, \delta_{2}\right)\right) \subset \eta^{+}\left(B_{E}^{u u}\left(q_{1}, \delta_{1}\right)\right) \cap \eta^{+}\left(B_{E}^{u u}\left(q_{2}^{\prime}, \delta_{1}\right)\right)=\emptyset .
$$

This completes the proof of (4.4).

By Theorem 3.10, there exists $0<\delta_{3}<\delta_{2}$ such that for all $q \in \Delta^{\prime}$ and all $q^{\prime} \in \mathcal{F}^{u u}(q)$ with $d_{E}\left(q, q^{\prime}\right)<\delta_{3}$ we have $d_{H}\left(q, q^{\prime}\right)<\delta$. For $q \in \Delta^{\prime}$, let

$$
H(q)=\eta^{+}\left(B_{E}\left(q, \delta_{3}\right)\right) \subset \eta^{+}(W(\delta)),
$$

Consider the collection of "balls" $\left\{H(q)\right.$ : $\left.q \in \Delta^{\prime}\right\}$. By (4.4) the sets $H(q)$ are pairwise disjoint viewed as subsets of $\mathcal{P} \mathcal{M F}$ (or alternatively the subsets $C$ one $(H(q)) \subset \mathcal{M F}$ intersect only at the origin). Also, by the definition of the Thurston measure $\nu$ and the compactness of $\mathcal{K}$, there exists a constant $c=c(\mathcal{K}, \delta)$ such that

$$
\bar{\nu}(H(q)) \geq c, \quad \text { for all } q \in \Delta^{\prime} .
$$

Hence,

$$
\bar{\nu}\left(\eta^{+}(W(\delta))\right) \geq \bar{\nu}\left(\bigcup_{q \in \Delta^{\prime}} H(q)\right)=\sum_{q \in \Delta^{\prime}} \bar{\nu}(H(q)) \geq c\left|\Delta^{\prime}\right|=c|\Delta| .
$$


Now (4.1) (with $W$ replaced by $W_{\alpha}$ ) follows from (4.3) and (4.7). Finally,

$$
\bar{\nu}\left(\eta^{+}(W(1))\right) \leq C(\delta, \mathcal{K}) \bar{\nu}\left(\eta^{+}(W(\delta))\right),
$$

since $d_{H}$ is equivalent to $d_{E}$.

The sets $K_{i}$ and $U_{i}$. Let $K_{1} \subset \mathcal{Q}^{1} \mathcal{M}_{g}$ be a compact set. (In our application, $K_{1}$ will be chosen disjoint from the multiple zero locus). Let $K_{3} \subset K_{2} \subset K_{1}$ and $1 \geq \delta>0$ be such that if $q \in K_{i}$ and $d_{H}\left(q, q^{\prime}\right)<c_{H} \delta$ then $q^{\prime} \in K_{i-1}$, where $c_{H}$ is as in Theorem 3.15 (a). We assume $\bar{\mu}\left(K_{3}\right)>(1 / 2)$, where $\bar{\mu}$ is the normalized Lebesgue measure on $\mathcal{Q}^{1} \mathcal{M}_{g}$. For $T_{0}>0$ let $U_{i}=U_{i}\left(T_{0}\right)$ be the set of $q \in \mathcal{Q}^{1} \mathcal{M}_{g}$ such that there exists $T>T_{0}$ so that

$$
\left|\left\{t \in[0, T]: g_{t} q \in K_{i}^{c}\right\}\right| \geq(1 / 2) T .
$$

Then, for all $T>T_{0}$ and all $q \notin U_{i}$,

$$
\left|\left\{t \in[0, T]: g_{t} q \in K_{i}^{c}\right\}\right|<(1 / 2) T .
$$

From the definition, we have $U_{1} \subset U_{2} \subset U_{3}$. By the ergodicity of the geodesic flow, for every $\theta>0$ there exists $T_{0}>0$ such that $\bar{\mu}\left(U_{3}\right)<\theta$.

Let $\mathcal{K}_{1}=\mathcal{K}$. We can choose compact subsets $\mathcal{K}_{0}, \mathcal{K}_{2}, \mathcal{K}_{3}$ of $\mathcal{T}_{g}$ such that for $0 \leq i \leq 2$, any $q \in \pi^{-1}\left(\mathcal{K}_{i}\right)$ and any $q^{\prime}$ on the same leaf of $\mathcal{F}^{u u}$ as $q$ with $d_{H}\left(q, q^{\prime}\right)<c_{H}$, we have $q^{\prime} \in \pi^{-1}\left(\mathcal{K}_{i+1}\right)$. By (the lower bound in) Theorem 3.10 and Lemma 3.9, each $\mathcal{K}_{i}$ is compact. Let $U_{i}^{\prime}=p^{-1}\left(U_{i}\right) \cap \pi^{-1}\left(\mathcal{K}_{i}\right)$, where $p$ is the natural map from $\mathcal{Q}^{1} \mathcal{T}_{g}$ to $\mathcal{Q}^{1} \mathcal{M}_{g}$. Note that $U_{1}^{\prime} \subset U_{2}^{\prime} \subset U_{3}^{\prime} \subset \mathcal{Q}^{1} \mathcal{T}_{g}$.

Lemma 4.2 Let $U_{i}^{\prime}, 1 \leq i \leq 3$ be as in the above paragraph. Then, for all $t>0$,

$$
\mathbf{m}\left(N b h d_{2}\left(\pi\left(g_{t} U_{1}^{\prime}\right) \cap \Gamma \mathcal{K}\right)\right) \leq C(\delta) e^{h t} \bar{\nu}\left(\eta^{+}\left(U_{2}^{\prime}\right)\right),
$$

and if $W(1)$ is defined as in Lemma 4.1 with $W=g_{t} U_{1}^{\prime} \cap \pi^{-1}(\Gamma \mathcal{K})$, then

$$
\bar{\nu}\left(\eta^{+}(W(1))\right) \leq C^{\prime}(\delta) e^{h t} \bar{\nu}\left(\eta^{+}\left(U_{2}^{\prime}\right)\right) .
$$

In particular (c.f. Theorem 2.2), for any $\epsilon>0$ is is possible to choose $T_{0}$ such that if $U_{1}$ is defined by 4.8) and $U_{1}^{\prime}=U_{1} \cap \pi^{-1}\left(\mathcal{K}_{1}\right)$ then for all $t>T_{0}$,

$$
\mathbf{m}\left(N b h d_{2}\left(\pi\left(g_{t} U_{1}^{\prime}\right)\right) \cap \Gamma \mathcal{K}\right) \leq \epsilon e^{h t},
$$

and for $W=g_{t} U_{1}^{\prime} \cap \pi^{-1}(\Gamma \mathcal{K})$ we have

$$
\bar{\nu}\left(\eta^{+}(W(1))\right) \leq \epsilon e^{h t} .
$$

Proof. We will apply Lemma 4.1 to the set $W=g_{t} U_{1}^{\prime} \cap \pi^{-1}(\Gamma \mathcal{K})$. We claim that $W(\delta) \subset g_{t} U_{2}^{\prime}$. Indeed, suppose $g_{t} q^{\prime} \in W(\delta)$. Then there exists $g_{t} q \in W$ with

$$
d_{H}\left(g_{t} q, g_{t} q^{\prime}\right)<\delta .
$$

Then, by Theorem 3.15 (a), for all $0 \leq s \leq t$,

$$
d_{H}\left(g_{s} q, g_{s} q^{\prime}\right)<c_{H} \delta
$$


Since $W=g_{t} U_{1}^{\prime}, g_{t} q \in W$ implies $q \in U_{1}^{\prime}$. Then, assuming $t>T_{0}$, for at least half the values of $s \in[0, t]$,

$$
g_{s} q \notin K_{1} .
$$

Then, (4.11) and the definition of $K_{2}$ imply that for the $s \in[0, t]$ for which (4.12) holds, $g_{s} q^{\prime} \notin K_{2}$. This implies $q^{\prime} \in U_{2}$. Since $d_{H}\left(q, q^{\prime}\right)<c_{H} \delta<c_{H}$ and $q \in \pi^{-1}(\mathcal{K})=\pi^{-1}\left(\mathcal{K}_{1}\right)$, we have $q^{\prime} \in \pi^{-1}\left(\mathcal{K}_{2}\right)$. Thus, $q^{\prime} \in U_{2} \cap \pi^{-1}\left(\mathcal{K}_{2}\right)=U_{2}^{\prime}$, and so $g_{t} q^{\prime} \in g_{t} U_{2}$. This implies the claim, and thus the first two statements of the Lemma.

The same argument as the proof of the claim shows that if $q \in U_{2}^{\prime}$ and $q^{\prime} \in \mathcal{F}^{s s}(q) \cap \pi^{-1}(\mathcal{K})$ with $d_{H}\left(q, q^{\prime}\right)<\delta$ then $q^{\prime} \in U_{3}^{\prime}$. This (together with Theorem 3.10) implies that there exists $C_{1}(\delta)$ such that

$$
\bar{\nu}\left(\eta^{+}\left(U_{2}^{\prime}\right)\right) \leq C_{1}(\delta) \bar{\mu}\left(U_{3}^{\prime}\right) .
$$

Hence if we choose $T_{0}>0$ so that $\bar{\mu}\left(U_{3}\right)<C(\delta) C_{1}(\delta) \epsilon$, then (4.9) follows. Similarly, if we choose $T_{0}>0$ so that in addition $\bar{\mu}\left(U_{3}\right)<C^{\prime}(\delta) C_{1}(\delta) \epsilon$, then (4.10) follows.

Proof of Theorem 2.7. Let $K^{\prime}=K_{1}$, and let $T_{0}, U_{1}$ and $U_{1}^{\prime}$ be as in Lemma 4.2 Then, for $R>T_{0}$, and $X \in \mathcal{K}$,

$$
B_{R}\left(X, \mathcal{K}, K^{\prime}\right) \subset \bigcup_{0 \leq t \leq R} \pi\left(g_{t} U_{1}^{\prime}\right) \cap \Gamma \mathcal{K} \quad \subset \bigcup_{n=0}^{\lfloor R\rfloor} \bigcup_{t \in[n, n+1]} \pi\left(g_{t} U_{1}^{\prime}\right) \cap \Gamma \mathcal{K},
$$

where $\lfloor x\rfloor$ denotes the integer part of $x$. Then,

$$
\mathbf{m}\left(\operatorname{Nbhd}_{1}\left(B_{R}\left(X, \mathcal{K}, K^{\prime}\right)\right)\right) \leq \sum_{n=0}^{\lfloor R\rfloor} \mathbf{m}\left(N b h d_{2}\left(\pi\left(g_{n} U_{1}^{\prime}\right) \cap \Gamma \mathcal{K}\right)\right) \leq C \epsilon \sum_{n=0}^{\lfloor R\rfloor} e^{h n},
$$

where we have used (4.9). Since $\epsilon$ is arbitrary, Theorem 2.7 follows.

Lemma 4.3 Suppose $V \subset \pi^{-1}(\mathcal{K})$ and $\delta^{\prime}>0$. Then, for $t$ sufficiently large (depending on $\mathcal{K}, V$ and $\left.\delta^{\prime}\right)$,

$$
\mathbf{m}\left(\operatorname{Nbhd}_{1}\left(\pi\left(g_{t} V\right) \cap \Gamma \mathcal{K}_{0}\right)\right) \leq C \bar{\nu}\left(\eta^{+}\left(V\left(\delta^{\prime}\right)\right)\right) e^{h t},
$$

where $C$ depends only on $\mathcal{K}$.

Proof. Let $Y=g_{t} V \cap \Gamma \mathcal{K}_{0}$. Choose $T_{0}$ so that (4.10) holds for $t>T_{0}$ and $\bar{\nu}\left(\eta^{+}\left(V\left(\delta^{\prime}\right)\right)\right)$ instead of $\epsilon$. Let $U_{1}, U_{1}^{\prime}$ and $W=g_{t} U_{1}^{\prime} \cap \pi^{-1}(\mathcal{K})$ be as in Lemma 4.2 so in particular, for $t>T_{0}$,

$$
\bar{\nu}\left(\eta^{+}(W(1))\right) \leq \bar{\nu}\left(\eta^{+}\left(V\left(\delta^{\prime}\right)\right)\right) e^{h t} .
$$

We claim that there exist $T_{1}>T_{0}$, depending only on $\mathcal{K}$ such that for $t>T_{1}$,

$$
Y(1)=W(1) \cup g_{t}\left(V\left(\delta^{\prime}\right)\right) .
$$

Indeed, if $g_{t} q \in Y(1)$ then by definition there exists $q^{\prime} \in V \subset \mathcal{K}$ with $d_{H}\left(g_{t} q, g_{t} q^{\prime}\right)<1$, and $q^{\prime}$ is on the same leaf of $\mathcal{F}^{u u}$ as $q$. We consider two cases: either $q^{\prime} \in U_{1}$ or $q^{\prime} \notin U_{1}$. If $q^{\prime} \in U_{1}$ then 
$q \in U_{1}^{\prime}=U_{1} \cap \mathcal{K}$, hence $g_{t} q^{\prime} \in g_{t} U_{1}^{\prime} \cap \Gamma \mathcal{K}=W$. Hence in this case, $g_{t} q \in W(1)$. If $q^{\prime} \notin U_{1}$, then by (4.8) and Theorem $3.15(\mathrm{~b})$,

$$
d_{H}\left(q, q^{\prime}\right)=d_{H}\left(g_{-t} g_{t} q, g_{-t} g_{t} q^{\prime}\right) \leq C e^{-c t} d_{H}\left(g_{t} q, g_{t} q^{\prime}\right) \leq C e^{-c t} .
$$

We choose $T_{1}>T_{0}$ so that $C e^{-c T_{1}}<\delta^{\prime}$. Thus, in this case, for $t>T_{1}, q \in V\left(\delta^{\prime}\right)$ and hence (4.14) follows.

Now, for $t>T_{1}$,

$$
\bar{\nu}\left(\eta^{+}(Y(1))\right) \leq \bar{\nu}\left(\eta^{+}(W(1))\right)+\bar{\nu}\left(\eta^{+}\left(g_{t}\left(V\left(\delta^{\prime}\right)\right)\right)\right) \leq 2 \bar{\nu}\left(\eta^{+}\left(V\left(\delta^{\prime}\right)\right)\right) e^{h t}
$$

where we have used (4.13). We now apply Lemma 4.1 with $\delta=1$. The lemma follows from (4.1).

Proof of Theorem 2.6. This follows immediately from Lemma 4.3, and the fact that we can choose an relatively open $V \subset \mathcal{K}$ and $\delta^{\prime}>0$ such that $V$ contains the intersection of $\mathcal{K}$ with the multiple zero locus, and $\bar{\nu}\left(\eta^{+}\left(V\left(\delta^{\prime}\right)\right)\right)$ is arbitrarily small, (see Theorem 2.2).

\section{Volume Asymptotics.}

In this section we prove Theorem 1.3 Let $F_{R}(X, Y)=\left|\Gamma \cdot Y \cap B_{R}(X)\right|$ be as in $\$ 2.6$ We need the following:

Theorem 5.1 Given $X \in \mathcal{T}_{g}$ there exists $C=C(X)$ such that for all $Y \in \mathcal{T}_{g}$,

$$
F_{R}(X, Y) \leq C(X) e^{h R} .
$$

\section{Notation.}

- Let $\mathcal{C}_{g}(\mathbb{N})$ be the set of isotopy classes of integral multicurves on a surface of genus $g$.

- Given $X, Y \in \mathcal{T}_{g}$ define

$$
M_{R}(X, Y)=\left\{\gamma \cdot Y, \gamma \in \Gamma \mid \gamma \cdot Y \in B_{R}(X)\right\},
$$

so that $F_{R}(X, Y)=\#\left(M_{R}(X, Y)\right)$.

Proof of Theorem 1.3 assuming Theorem 5.1, By definition of $F_{R}$,

$$
\mathbf{m}\left(B_{R}(X)\right)=\int_{\mathcal{M}_{g}} F_{R}(X, Y) d \mathbf{m}(Y)
$$

We multiply both sides by $e^{-h R}$ and take the limit at $R \rightarrow \infty$. By Theorem 5.1 we can apply the bounded convergence theorem to take the limit inside the integral. Now the theorem follows from Theorem 1.2 .

A similar argument yields the following: 
Theorem 5.2 Suppose $X \in \mathcal{T}_{g}$, and $\mathcal{U} \subset S(X)$. Then, as $R \rightarrow \infty$,

$$
\mathbf{m}\left(B_{R}(X) \cap \operatorname{Sect}_{\mathcal{U}}(X)\right) \sim \frac{1}{h \mathbf{m}\left(\mathcal{M}_{g}\right)} e^{h R} \Lambda(Y) \int_{\mathcal{U}} \lambda^{-}(q) d s_{X}(q) .
$$

In the rest of this section we prove Theorem 5.1. Along the way, we prove Theorem 2.4

Estimating extremal lengths. Consider the Dehn-Thurston parameterization HP92 of the set of multicurves

$$
D T: \mathcal{C}_{g}(\mathbb{N}) \rightarrow\left(\mathbb{Z}_{+} \times \mathbb{Z}\right)^{3 g-3}
$$

defined by

$$
D T(\beta)=\left(i\left(\beta, \alpha_{i}\right), \operatorname{tw}\left(\beta, \alpha_{i}\right)\right)_{i=1}^{3 g-3},
$$

where $i(\cdot, \cdot)$ denotes the geometric intersection number and $\operatorname{tw}\left(\beta, \alpha_{i}\right)$ is the twisting parameter of $\beta$ around $\alpha_{i}$. See HP92 for more details. By a theorem of Bers, we can choose a constant $C_{g}$ depending only on $g$ such that for any surface $Y \in \mathcal{T}_{g}$ there exists a pants decomposition $\mathcal{P}=\left\{\alpha_{1}, \ldots, \alpha_{3 g-3}\right\}$ on $Y$ such that for $1 \leq i \leq 3 g-3$

$$
\operatorname{Ext}_{\alpha_{i}}(Y) \leq C_{g}^{2} .
$$

We call such a pants decomposition a bounded pants decomposition for $Y$. The following result is proved in Mi96] (see Theorem 5.1, and equation (4.3)):

Theorem 5.3 (Minsky) Suppose $Y \in \mathcal{T}_{g}$, and let $\mathcal{P}=\mathcal{P}(Y)=\left\{\alpha_{1}, \ldots, \alpha_{3 g-3}\right\}$ be any bounded pants decomposition on $Y$. Then given a simple closed curve $\beta, \operatorname{Ext}_{\beta}(Y)$ is bounded from above and below by

$$
\max _{1 \leq j \leq 3 g-3}\left[\frac{i\left(\beta, \alpha_{j}\right)^{2}}{\operatorname{Ext}_{\alpha_{j}}(Y)}+\operatorname{tw}^{2}\left(\beta, \alpha_{j}\right) \operatorname{Ext}_{\alpha_{j}}(Y)\right],
$$

up to a multiplicative constant depending only on $g$.

Theorem 5.3 gives a bound on the the Dehn-Thurston coordinates of a simple closed curve in terms of its extremal length.

Remark. The definition of the twist used in equation (4.3) in Mi96 is different from the definition we are using here. We follow the definition used in HP92. Given a connected simple closed curve $\alpha$ on $\Sigma_{g}$, let $h_{\alpha} \in \Gamma$ denote the right Dehn twist around $\alpha$. Then in terms of our notation,

$$
\operatorname{tw}\left(h_{\alpha}^{r}(\beta), \alpha\right)=\operatorname{tw}(\beta, \alpha)+r \cdot i(\beta, \alpha) .
$$

Corollary 5.1 Let $\alpha=\left\{\alpha_{1}, \ldots, \alpha_{3 g-3}\right\}$ be bounded pants decomposition of $X \in \mathcal{T}_{g}$. Then there is a constant $c_{1}>0$ such that for any simple closed curve $\beta$ on $\Sigma_{g}$,

$$
\operatorname{tw}\left(\beta, \alpha_{i}\right) \leq c_{1} \cdot \frac{\sqrt{\operatorname{Ext}_{\beta}(X)}}{\sqrt{\operatorname{Ext}_{\alpha_{i}}(X)}},
$$

and

$$
i\left(\beta, \alpha_{i}\right) \leq \sqrt{\operatorname{Ext}_{\beta}(X)} \cdot \sqrt{\operatorname{Ext}_{\alpha_{i}}(X)} \leq c_{1} \sqrt{\operatorname{Ext}_{\beta}(X)} .
$$


Estimating the number of multicurves. Define

$$
E(Y, L)=\#\left|\left\{\alpha \in \mathcal{C}_{g}(\mathbb{N}) \mid \sqrt{\operatorname{Ext}_{\alpha}(Y)} \leq L\right\}\right|
$$

Fix $\epsilon_{0}>0$ small enough such that if $\alpha$ and $\beta$ on $Y \in \mathcal{T}_{g}$ satisfy $\operatorname{Ext}_{\alpha}(Y) \leq \epsilon_{0}^{2}$ and $\operatorname{Ext}_{\beta}(Y) \leq C_{g}^{2}$ then $i(\alpha, \beta)=0$. Note that any bounded pants decomposition $\mathcal{P}$ on $Y$ includes all simple closed curves of extremal length $\leq \epsilon_{0}^{2}$ on $Y$.

Let

$$
G(Y)=1+\prod_{\operatorname{Ext}_{\gamma}(Y) \leq \epsilon_{0}^{2}} \frac{1}{\sqrt{\operatorname{Ext}_{\gamma}(Y)}},
$$

and the product ranges over all simple closed curves $\gamma$ on the surface $Y$ with $\operatorname{Ext}_{\gamma}(Y) \leq \epsilon_{0}^{2}$.

Using Theorem 5.3, we obtain the following:

Theorem 5.4 There exists a constant $C>0$ such that for every $Y \in \mathcal{T}_{g}$ and $L>0$ we have

$$
E(Y, L) \leq C \cdot G(Y) \cdot L^{6 g-6} .
$$

Moreover, for any $Y$, if $1 / L$ is bounded by an absolute constant times the square root of the extremal length of the shortest curve on $Y$, then

$$
E(Y, L) \leq C L^{6 g-6}
$$

Sketch of the proof. In order to use the bound given by equation (5.1), first we fix a bounded pants decomposition

$$
\mathcal{P}=\left\{\alpha_{1}, \ldots, \alpha_{3 g-3}\right\}
$$

on $Y$. Note that, this pants decomposition should include all small closed curves on $Y$. Let $m_{i}=$ $i\left(\beta, \alpha_{i}\right), t_{i}=\operatorname{tw}\left(\beta, \alpha_{i}\right)$, and $s_{i}=\sqrt{\operatorname{Ext}_{\alpha_{i}}(Y)}$. So by equation (5.1), $\operatorname{Ext}_{\beta}(Y) \leq L^{2}$ implies that $\frac{m_{i}}{s_{i}}+\left|t_{i}\right| s_{i}=O(L)$. We use the following elementary lemma:

Lemma 5.5 For $s>0$, define $A_{s}(L)$ by

$$
A_{s}(L)=\left\{(a, b) \mid a, b \in \mathbb{Z}_{+}, a \cdot s+\frac{b}{s} \leq L\right\} \subset \mathbb{Z}_{+} \times \mathbb{Z}_{+} .
$$

Then for any $L>0,\left|A_{s}(L)\right| \leq 4 \max \{s, 1 / s\} \cdot L^{2}$. For $L>\max \left\{s, \frac{1}{s}\right\}$, we have $A_{s}(L) \leq 4 L^{2}$.

Now applying the preceding lemma to the $s_{i}$, and using the Dehn-Thurston parameterization of multicurves, we get

$$
E(Y, L) \leq \prod_{i=1}^{3 g-3}\left|A_{s_{i}}(L)\right| \leq C G(Y) L^{6 g-6} .
$$

Proof of Theorem 2.4. This follows from the second part of Theorem 5.4. Recall (see \$2.1) that the extremal length can be extended continuously to a map

$$
\text { Ext }: \mathcal{M F} \times \mathcal{T}_{g} \rightarrow \mathbb{R}_{+}
$$


such that $\operatorname{Ext}_{t \lambda}(X)=t^{2} \operatorname{Ext}_{\lambda}(X)$. Also the space $\mathcal{M F}$ has a piecewise linear integral structure, and elements of $\mathcal{S}_{g}$ are in one to one correspondence with the integral points. Hence,

$$
\Lambda(X)=\operatorname{Vol}\left\{\eta \in \mathcal{M F} \mid \sqrt{\operatorname{Ext}_{\eta}(X)} \leq 1\right\}=\frac{\operatorname{Vol}\left\{\eta \in \mathcal{M F} \mid \sqrt{\operatorname{Ext}_{\eta}(X)} \leq L\right\}}{L^{6 g-6}}=\lim _{L \rightarrow \infty} \frac{E(x, L)}{L^{6 g-6}},
$$

where to justify the last equality use the fact that for any as $L \rightarrow \infty$ the number of lattice points in the dilation $L A$ is asymptotic to the volume of $L A$, where $A=\left\{\eta \in \mathcal{M F} \mid \sqrt{\operatorname{Ext}_{\eta}(X)} \leq 1\right\}$.

On the other hand, by the second assertion in Theorem 5.4 when $L$ is big enough $E(Y, L) \leq$ $C L^{6 g-6}$. Therefore, $\Lambda(Y) \leq C$, where the bound does not depend on $Y$.

Remark. Let $p: \mathcal{T}_{g} \rightarrow \mathcal{M}_{g}$ be the natural projection. Fix a compact subset $\mathcal{K} \subset \mathcal{M}_{g}$. For simplicity, we will also denote $p^{-1}(\mathcal{K}) \subset \mathcal{T}_{g}$ by $\mathcal{K}$. If $\mathcal{P}=\left\{\alpha_{i}\right\}$ is a bounded pants decomposition on $X \in \mathcal{K}$ then for any $1 \leq i \leq 3 g-3$,

$$
\frac{1}{C_{\mathcal{K}}} \leq \sqrt{\operatorname{Ext}_{\alpha_{i}}(X)} \leq C_{\mathcal{K}}
$$

where $C_{\mathcal{K}}$ is a constant which only depends on $\mathcal{K}$. Therefore, by Corollary [5.1, for the elements in the thick part of the Teichmüller space, the Dehn-Thurston coordinates of a simple closed curve are bounded by its extremal length. More precisely, if $\alpha=\left\{\alpha_{1}, \ldots, \alpha_{3 g-3}\right\}$ be bounded pants decomposition of $X \in \mathcal{K}$. then there is a constant $c_{1}>0$, depending only on $\mathcal{K}$, such that for any simple closed curve $\beta$ on $\Sigma_{g}$,

$$
\operatorname{tw}\left(\beta, \alpha_{i}\right) \leq c_{1} \cdot \sqrt{\operatorname{Ext}_{\beta}(X)}, \quad i\left(\beta, \alpha_{i}\right) \leq c_{1} \cdot \sqrt{\operatorname{Ext}_{\beta}(X)} .
$$

Proposition 5.6 There exists a constant $C_{3}$ depending only on $\mathcal{K}$ such that for every $X \in \mathcal{K}$, $Y \in \mathcal{T}_{g}$ and $R>0$ we have

$$
F_{R}(X, Y) \leq C_{3} E\left(Y, e^{R}\right) .
$$

Our goal is to assign to any point $Z=\gamma \cdot Y \in M_{R}(X, Y)$ a unique integral multicurve $\beta_{Z} \in$ $E\left(Y, e^{R}\right)$. This would imply that

$$
F_{R}(X, Y) \leq C e^{(6 g-6) R} .
$$

(Note that we are assuming that $X$ is in the compact part of $\mathcal{T}_{g}$ and $d(X, Y)<R$; this implies that the shortest curve on $Y$ has extremal length at least constant times $e^{-2 R}$, hence we may use the second statement of Theorem [5.4)

The most natural candidate for $\beta_{Z}$ is $\gamma^{-1} \alpha$ where $\alpha$ is a fixed pants decomposition of $X$. However this correspondence is not one to one. Therefore, we need to modify the construction.

Given $Z=\gamma \cdot Y \in M_{R}(X, Y)$, and $\alpha \in \mathcal{S}_{g}$ we have

$$
\frac{\sqrt{\operatorname{Ext}_{\alpha}(Z)}}{\sqrt{\operatorname{Ext}_{\alpha}(X)}} \leq e^{R}
$$

So by setting $L=e^{R}$, and $\beta=\gamma^{-1}(\alpha)$ we have

$$
\sqrt{\operatorname{Ext}_{\beta}(Y)} \leq L \cdot \sqrt{\operatorname{Ext}_{\alpha}(X)} .
$$


Given $Z=\gamma \cdot Y \in M_{R}(X, Y)$, let $\alpha(Z)_{i}=\gamma^{-1} \alpha_{i} \in \mathcal{S}_{g}$. Then from (5.4) we get

$$
\sqrt{\operatorname{Ext}_{\alpha(Z)_{i}}(Y)} \leq C_{\mathcal{K}} \cdot L
$$

Moreover, for $Z_{1}=\gamma_{1} Y, Z_{2}=\gamma_{2} Y \in \Gamma \cdot Y$, we have $\alpha\left(Z_{1}\right)=\alpha\left(Z_{2}\right)$ if and only if there are $r_{1}, \ldots r_{3 g-3} \in \mathbb{Z}$ such that

$$
\gamma_{1}=h_{\alpha_{1}}^{r_{1}} \cdots h_{\alpha_{3 g-3}}^{r_{3 g-3}} \cdot \gamma_{2}
$$

Moreover, we have:

Lemma 5.7 Let $\alpha=\left\{\alpha_{1}, \ldots, \alpha_{3 g-3}\right\}$ be a bounded pants decomposition on $X$ and suppose $Y_{0} \in$ $B_{R}(X)$. If $h_{\alpha_{1}}^{r_{1}} \cdots h_{\alpha_{3 g-3}}^{r_{3 g-3}}\left(Y_{0}\right) \in B_{R}(X)$ then for $1 \leq i \leq 3 g-3$

$$
\left|r_{i}\right| \cdot \sqrt{\operatorname{Ext}_{\alpha_{i}}\left(Y_{0}\right)} \leq C_{2} \frac{e^{R}}{\sqrt{\operatorname{Ext}_{\alpha_{i}}(X)}}
$$

Here $C_{2}$ is a constant independent of $X$ and $Y_{0}$.

Sketch of the proof of Lemma 5.7. Let $s_{i}=\sqrt{\operatorname{Ext}_{\alpha_{i}}\left(Y_{0}\right)}$, and $L=e^{R}$. The key point in the proof is Lemma 6.3 in [Mi96]. From this lemma, for any $i$, there exists a multicurve $\beta_{i}$ of extremal length $b_{i}^{2}=\operatorname{Ext}_{\beta_{i}}\left(Y_{0}\right)$ such that

$$
i\left(\beta_{i}, \alpha_{i}\right) \geq c s_{i} \cdot b_{i}
$$

Since $d\left(X, Y_{0}\right) \leq R, \sqrt{\operatorname{Ext}_{\beta_{i}}(X)} \leq b_{i} \cdot L$. Also the assumption

$$
d\left(h_{\alpha_{1}}^{-r_{1}} \cdots h_{\alpha_{3 g-3}}^{-r_{3 g-3}}(X), Y_{0}\right) \leq R
$$

implies that

$$
\sqrt{\left.\operatorname{Ext}_{h_{\alpha_{1}}^{r_{1}} \ldots h_{\alpha_{3 g-3}}^{r_{3 g-3}}\left(\beta_{i}\right)}(X)\right)}=\sqrt{\operatorname{Ext}_{\beta_{i}}\left(h_{\alpha_{1}}^{-r_{1}} \cdots h_{\alpha_{3 g-3}}^{-r_{3 g-3}}(X)\right)} \leq b_{i} \cdot L
$$

Then

1. Since $\sqrt{\operatorname{Ext}_{\beta_{i}}(X)} \leq b_{i} \cdot L$, Corollary 5.1 implies that the twist and intersection coordinates of the curve $\beta_{i}$ are bounded by a multiple of $b_{i} \cdot L$; in particular

$$
\left|\operatorname{tw}\left(\beta_{i}, \alpha_{i}\right)\right|=O\left(\frac{b_{i} \cdot L}{\sqrt{\operatorname{Ext}_{\alpha_{i}}(X)}}\right) .
$$

2. Similarly, by equation (5.5), applying Corollary 5.1 for $h_{\alpha_{1}}^{r_{1}} \cdots h_{\alpha_{3 g-3}}^{r_{3 g-3}}\left(\beta_{i}\right)$ on $X$ with respect to $\left\{\alpha_{i}\right\}$ implies that

$$
\left|\operatorname{tw}\left(h_{\alpha_{1}}^{r_{1}} \cdots h_{\alpha_{3 g-3}}^{r_{3 g-3}}\left(\beta_{i}\right), \alpha_{i}\right)\right|=O\left(\frac{b_{i} \cdot L}{\sqrt{\operatorname{Ext}_{\alpha_{i}}(X)}}\right) .
$$

3. On the other hand, by the definition (see equation (5.2) ) we have

$$
\begin{gathered}
\left|\operatorname{tw}\left(h_{\alpha_{1}}^{r_{1}} \ldots h_{\alpha_{3 g-3}}^{r_{3 g-3}}\left(\beta_{i}\right), \alpha_{i}\right)\right|=\left|r_{i} \cdot i\left(\alpha_{i}, \beta_{i}\right)+\operatorname{tw}\left(\beta_{i}, \alpha_{i}\right)\right| \geq \\
\geq\left|r_{i}\right| \cdot i\left(\alpha_{j}, \beta_{i}\right)-\left|\operatorname{tw}\left(\beta_{i}, \alpha_{i}\right)\right| .
\end{gathered}
$$


So we have

$$
\left|r_{i}\right| \cdot s_{i} \cdot b_{i} \leq \frac{1}{c}\left|r_{i}\right| \cdot i\left(\beta_{i}, \alpha_{i}\right) \leq C_{2} L \cdot b_{i} \Longrightarrow\left|r_{i}\right| \cdot s_{i} \leq \frac{C_{2} L}{\sqrt{\operatorname{Ext}_{\alpha_{i}}(X)}} .
$$

Remark. As a result, if the assumption of Lemma 5.7 holds and $X \in \mathcal{K}$ then

$$
\left|r_{i}\right| \cdot \sqrt{\operatorname{Ext}_{\alpha_{i}}\left(Y_{0}\right)} \leq C e^{R}
$$

where $C$ is a constant which only depends on $\mathcal{K}$.

We remark that for any two disjoint simple closed curves $\eta_{1}$ and $\eta_{2}$, and $m_{1}, m_{2}>0$, we have, by the definition of extremal length,

$$
\sqrt{\operatorname{Ext}_{m_{1} \cdot \eta_{1}}(X)}+\sqrt{\operatorname{Ext}_{m_{2} \cdot \eta_{2}}(X)} \geq \sqrt{\operatorname{Ext}_{m_{1} \cdot \eta_{1}+m_{2} \cdot \eta_{2}}(X)} .
$$

Corollary 5.2 If the assumption of Lemma[5.7 holds and $X \in K$ then for $\widehat{\alpha}=\sum_{i=1}^{3 g-3}\left|r_{i}\right| \cdot \alpha_{i}$ we have

$$
\sqrt{\operatorname{Ext}_{\widehat{\alpha}}\left(Y_{0}\right)} \leq C_{3} e^{R}
$$

Therefore, $\widehat{\alpha}$ also defines a multicurve of extremal length bounded by $e^{2 R}$. This completes the proof of Proposition 5.6, and thus in view of Theorem 5.4 the proof of Theorem 5.1]

\section{A Appendix: Proof of Theorem 2.2.}

In this section, $\mathcal{Q}^{*} \mathcal{T}_{g}$ denotes the space of non-zero quadratic differentials on marked compact surfaces of genus $g$, without restriction on area.

A local coordinate system near the multiple zero locus. Near the multiple zero locus, the coordinate system given by the period map (see Lemma 3.8) is singular. Instead we use an alternative coordinate system from HuMas79.

Suppose $q_{0} \in \mathcal{Q}^{*} \mathcal{T}_{g}$ has multiple zeroes, say $w_{1}, \ldots, w_{m}$. Let $m_{i}$ be the multiplicity of $q_{0}$ at $w_{i}$. Then, by [HuMas79, Proposition 3.1], for $1 \leq i \leq m$ there exists a choice of local coordinate $z_{i}$, mapping $w_{i}$ to 0 , such that any $q \in \mathcal{Q}^{*} \mathcal{T}_{g}$ near $q_{0}$, on a neighborhood of $w_{i}$, has the form:

$$
q=\left(z_{i}^{m_{i}}+\sum_{j=0}^{m_{i}-2} a_{i j} z_{i}^{j}\right)\left(d z_{i}\right)^{2} .
$$

The coordinates $z_{i}$ are uniquely determined by $q$. Fix some $\delta>0$, and let $w_{i}^{\prime}$ be the point corresponding to $z_{i}=\delta$. For $q \in \mathcal{Q}^{*} \mathcal{T}_{g}$ near $q_{0}$ let $\tilde{X}$ denote the canonical double cover which makes the foliations corresponding to $q$ orientable, and let $\sigma$ be the involution of $\tilde{X}$ so that $\tilde{X} / \sigma$ is the surface $X$ (with the flat structure given by $q$ ). Let $\Sigma_{\delta}=\sigma^{-1}\left(w_{1}^{\prime}, \ldots, w_{m}^{\prime}\right.$ ), and let $H$ denote the relative homology group $H_{1}\left(\tilde{X}, \Sigma_{\delta}, \mathbb{Z}\right)$. Let $H_{\text {odd }}$ denote the odd part of $H$ under the action of the involution $\sigma$. Let $\gamma_{1}, \ldots, \gamma_{m}$ be a integral basis for $H_{\text {odd }}$. Let

$$
\lambda_{i}=\int_{\gamma_{i}} \sqrt{q} .
$$


Suppose $m_{i}$ is even. Let $w_{i j}, 1 \leq j \leq m_{i}$ denote the zeroes of $q$ which tend to $w_{i}$ as $q \rightarrow q_{0}$. Let $\eta_{i}$ be a fixed small circle in the coordinates $z_{i}$, centered at $z_{i}=0$ (i.e. $w_{i}$ ). Then if $q$ is sufficiently close to $q_{0}, \eta_{i}$ separates all the $w_{i j}$ from the rest of the surface. Let $b_{i}=\int_{\eta_{i}} \sqrt{q}$. Then, a residue calculation shows that

$$
b_{i}=a_{\frac{m_{i}}{2}-1}+\text { some polynomial in } a_{i j}, m_{i}-2 \geq j \geq \frac{m_{i}}{2} .
$$

Proposition A.1 (Hubbard-Masur) If $q_{0}$ is not a square of an Abelian differential, then there exists a neighborhood $U \subset \mathcal{Q}^{*} \mathcal{T}_{g}$ of $q_{0}$ such that the $a_{i j}$ and the $\lambda_{i}$ are smooth local coordinates on $U$. If $q_{0}$ is the square of an Abelian differential, then there exists a neighborhood $U$ of $q_{0}$ such that under the constraint $\sum_{i} b_{i}=0$, the $a_{i j}$ and the $\lambda_{i}$ are local coordinates on $U$.

In both cases, near $q_{0}$, the natural projection map $\pi: \mathcal{Q}^{*} \mathcal{T}_{g} \rightarrow \mathcal{T}_{g}$ is a submersion in these coordinates.

Proof. See [HuMas79, Proposition 4.7].

The constraint $\sum_{i} b_{i}=0$ appears because in the case when $q_{0}$ is the square of an Abelian differential, the part of the surface which is outside all the circles $\eta_{i}$ has an orientable foliation. In that case, in view of A.1 , we may drop $a \frac{m_{i}}{2}-1$ for some $i$ from the coordinate system.

We introduce a covering of $\mathcal{Q}^{*} \mathcal{T}_{g}$ by open sets $U_{\alpha}$, such that each $U_{\alpha}$ is the neighborhood $U$ of Proposition A.1. for some $q_{0} \in \mathcal{Q}^{*} \mathcal{T}_{g}$. We may assume that the $U_{\alpha}$ are invariant under the operation of multiplying the quadratic differential by a real number. We also assume that covering is uniformly locally finite on compact sets, i.e. that for any compact set $\mathcal{K} \in \mathcal{T}_{g}$, there exist a number $N$ depending only on $\mathcal{K}$ such that each point in $\hat{\pi}^{-1}(\mathcal{K})$ belongs to at most $N$ sets $U_{\alpha}$.

Let $\psi_{\alpha}$ be a partition of unity subordinate to $U_{\alpha}$. For $q, q^{\prime} \in U_{\alpha}$, let $\left\{a_{i j}, \lambda_{i}\right\}$ be the coordinates of $q$, and $\left\{a_{i j}^{\prime}, \lambda_{i}^{\prime}\right\}$ be the coordinates of $q^{\prime}$. Let $D_{\alpha}\left(q, q^{\prime}\right)=\sum_{i j}\left|a_{i j}-a_{i j}^{\prime}\right|+\sum_{i}\left|\lambda_{i}-\lambda_{i}^{\prime}\right|$. For $q$ and $q^{\prime}$ sufficiently close so that they belong to the same $U_{\alpha}$, let $D\left(q, q^{\prime}\right)=\sum_{\alpha} \psi_{\alpha} D_{\alpha}\left(q, q^{\prime}\right)$.

Recall $d_{\mathcal{T}}(X, Y)$ denotes the Teichmüller distance between $X$ and $Y$, and $S(X)$ denotes the sphere of unit area quadratic differentials which are holomorphic at $X$.

Lemma A.2 Suppose $X, Y$ in $\mathcal{T}_{g}$ are sufficiently close and belong to a compact set $\mathcal{K}$. Then, there exist $c>1$ depending only on $\mathcal{K}$ such that

$$
c^{-1} \inf _{q \in S(X)} D\left(q, \pi^{-1}(Y)\right) \leq d_{\mathcal{T}}(X, Y) \leq c \sup _{q \in S(X)} D\left(q, \pi^{-1}(Y)\right) .
$$

Proof. Let $K$ be the pullback of $\mathcal{K}$ from $\mathcal{T}_{g}$ to $\mathcal{Q}^{1} \mathcal{T}_{g}$. Then $K$ is also compact. Near any $q \in K$, by Proposition A.1, the map $\pi: \mathcal{Q}^{*} \mathcal{T}_{g} \rightarrow \mathcal{T}_{g}$ is a submersion, and therefore can be approximated by a linear map, for which A.2. is immediate. The rest follows by compactness.

Let $\beta_{\alpha}$ be the measure on $U_{\alpha} \subset \mathcal{Q}^{*} \mathcal{T}_{g}$ given by $\prod_{i, j} d a_{i j} \prod_{i} d \lambda_{i}$. Let $\beta=\sum_{\alpha} \psi_{\alpha} \beta_{\alpha}$.

For $V \subset \pi^{-1}(X)$, let $V^{*} \subset \mathcal{Q}^{*} \mathcal{T}_{g}$ denote the set of all quadratic differentials which have the same horizontal foliation as some $q \in V$.

Lemma A.3 For every $\delta>0$, any $X \in \mathcal{T}_{g}$ and any $q_{0} \in \mathcal{Q}^{*} \mathcal{T}_{g}$ there exists an open subset $V$ of $\pi^{-1}(X)$ containing $q_{0}$, such that for all sufficiently small $\epsilon>0$,

$$
\beta\left(V^{*} \cap \pi^{-1}(B(X, \epsilon))\right)<\delta \epsilon^{6 g-6} .
$$


Proof. Fix $\delta^{\prime}>0$. Recall that by a horosphere (e.g. leaf of $\mathcal{F}^{s s}$ ) in $\mathcal{Q}^{*} \mathcal{T}_{g}$ we mean the set of quadratic differentials with a fixed horizontal foliation. Since the foliation by horospheres is continuous, there exists a neighborhood $V$ of $q_{0} \in \pi^{-1}(X)$ such that $V \subset B_{D}\left(q_{0}, \delta^{\prime}\right)$, where $B_{D}$ denotes a ball in the metric $D$. Then, for $\epsilon>0$ sufficiently small, and in view of Lemma A.2 there exist constants $C_{1}, C_{2}$ such that

$$
\pi^{-1}\left(V^{*} \cap B(X, \epsilon)\right) \subset\left\{q \in \mathcal{Q}^{*} \mathcal{T}_{g}: \quad D\left(q, B_{D}\left(q_{0}, C_{2} \delta^{\prime}\right) \cap \pi^{-1}(X)\right)<C_{1} \epsilon\right\} .
$$

Since $\pi^{-1}(X)$ is smooth, the $\beta$-measure of the set on the right of (A.3) is $\epsilon^{6 g-6} c\left(\delta^{\prime}\right)$, where $c\left(\delta^{\prime}\right) \rightarrow 0$ as $\delta^{\prime} \rightarrow 0$. This implies the lemma.

Proposition A.4 The measure $\mu$ on $\mathcal{Q}^{*} \mathcal{T}_{g}$ is absolutely continuous with respect to $\beta$, and for any compact subset $\mathcal{K}$ of $\mathcal{T}_{g}$ there exists a constant $C$ such that for all $q \in \pi^{-1}(\mathcal{K}),\left|\frac{d \mu}{d \beta}(q)\right|<C$. Also, away from the multiple zero locus, $\left|\frac{d \mu}{d \beta}(q)\right|$ is a smooth non-vanishing function of $q$.

Remark. It is also possible to show that there exists a constant $C^{\prime}$ depending only on $\mathcal{K}$ such that for all $q \in \pi^{-1}(\mathcal{K})$, one has $C^{\prime}<\left|\frac{d \mu}{d \beta}(q)\right|$. Since we do not need this, we will omit the proof.

Proof of Theorem 2.2, assuming Proposition A.4. By Lemma A.2, $\beta\left(\pi^{-1}(B(X, \epsilon))\right)=$ $O\left(\epsilon^{6 g-6}\right)$. Therefore, by Proposition A.4 there exist $0<c_{1}<c_{2}$ such that

$$
c_{1} \epsilon^{6 g-6}<\mu\left(\pi^{-1}(B(X, \epsilon))\right)<c_{2} \epsilon^{6 g-6} .
$$

Now,

$$
d s_{X}(V)=\lim _{\epsilon \rightarrow 0} \frac{\mu\left(V^{*} \cap \pi^{-1}(B(X, \epsilon))\right)}{\mu\left(\pi^{-1}(B(X, \epsilon))\right)} \leq \limsup _{\epsilon \rightarrow 0} \frac{C \beta\left(V^{*} \cap \pi^{-1}(B(X, \epsilon))\right)}{\mu\left(\pi^{-1}(B(X, \epsilon))\right)} \leq \frac{C \delta \epsilon^{6 g-6}}{c_{1} \epsilon^{6 g-6}} .
$$

where for the first inequality we used Proposition A.4, and for the last estimate we used Lemma A.3 and (A.4). Since $\delta$ is arbitrary, the theorem follows.

The rest of the section will consist of the proof of Proposition A.4 To simplify notation, we work with one zero of $q_{0}$ at a time. We write:

$$
z^{m}+\sum_{i=0}^{m-1} a_{i} z^{i}=\prod_{j=1}^{m}\left(z-z_{j}\right) .
$$

Then the $a_{i}$ are symmetric polynomials in the $z_{j}$. It is well known that the Jacobian of the map from the zeroes $z_{j}$ 's to the coefficients $a_{i}$ 's is the Vandermonde determinant. In other words, if we use the notation $\frac{\partial\left(y_{1}, \ldots, y_{n}\right)}{\partial\left(x_{1}, \ldots, x_{n}\right)}$ for the Jacobian determinant of the matrix $\left\{\frac{\partial y_{i}}{\partial x_{j}}\right\}_{1 \leq i, j \leq n}$, we have:

$$
\frac{\partial\left(a_{0}, \ldots, a_{m-1}\right)}{\partial\left(z_{1}, \ldots, z_{m}\right)}=\prod_{i<j}\left(z_{i}-z_{j}\right) .
$$

(This follows from comparing degrees and noting the anti-symmetry of the determinant). 
We choose a basis for the homology relative to the zeroes. This amounts to choosing a spanning subtree $T$ from the complete graph connecting the zeroes $z_{j}$. We may choose the tree in such a way that for any $z_{i}$ and $z_{j},\left|z_{i}-z_{j}\right|$ is within a multiplicative constant (depending only on $m$ ) of the length of the path in $T$ connecting $z_{i}$ and $z_{j}$. Let the (oriented) edges of $T$ be $e_{1}, \ldots, e_{m-1}$. Let $e_{k}^{+}$ be the zero at the head of $e_{k}$, and $e_{k}^{-}$the zero at the tail of $e_{k}$. We write $\vec{e}_{k}=e_{k}^{+}-e_{k}^{-}$(so $\vec{e}_{k} \in \mathbb{C}$ ).

In our setting, we need to restrict to $a_{m-1}=0$. Note that $a_{m-1}=z_{1}+\cdots+z_{m}$. It follows that

$$
\frac{\partial\left(a_{0}, \ldots, a_{m-2}\right)}{\partial\left(\vec{e}_{1}, \ldots, \vec{e}_{m-1}\right)}=\frac{\partial\left(a_{0}, \ldots, a_{m-1}\right)}{\partial\left(\vec{e}_{1}, \ldots, \vec{e}_{m-1}, a_{m-1}\right)}=\frac{\partial\left(a_{0}, \ldots, a_{m-1}\right)}{\partial\left(z_{1}, \ldots, z_{m}\right)} \frac{\partial\left(z_{1}, \ldots, z_{m}\right)}{\partial\left(\vec{e}_{1}, \ldots, \vec{e}_{m-1}, a_{m-1}\right)}=\prod_{i<j}\left(z_{i}-z_{j}\right),
$$

where we have used (A.5).

If $z_{j}$ is a zero, and $e$ is an edge of $T$, let

$$
d_{+}\left(z_{j}, e\right)=\max \left(\left|z_{j}-e^{-}\right|,\left|z_{j}-e^{+}\right|\right) .
$$

We will need the following combinatorial lemma:

\section{Lemma A.5}

$$
\prod_{e \in T} \prod_{p=1}^{m} d_{+}\left(z_{p}, e\right)^{1 / 2} \leq C \prod_{i<j}\left|z_{i}-z_{j}\right|,
$$

where $C$ depends only on $m$.

Proof of Lemma A.5. Let $e^{\prime}$ be the longest edge of $T$. If we cut along $e^{\prime}$, we separate the tree into two subtrees $T_{1}$ and $T_{2}$ say of size $m_{1}$ and $m_{2}$. If $z_{i} \in T_{1}$ and $z_{j} \in T_{2}$, then by the assumption on $T,\left|z_{i}-z_{j}\right|$ is comparable to the length of the path in the tree connecting $z_{i}$ to $z_{j}$. This path contains $e^{\prime}$, which is by assumption the longest edge in the tree. Therefore, if $z_{i} \in T_{1}$ and $z_{j} \in T_{2}$ then $\left|z_{i}-z_{j}\right|$ is within a multiplicative constant of $e^{\prime}$. Hence, the left hand side of A.7 is within a multiplicative constant of

$$
\left|e^{\prime}\right|^{m_{1} m_{2}} \prod_{\substack{i<j \\ z_{i}, z_{j} \in T_{1}}}\left|z_{i}-z_{j}\right| \prod_{\substack{i<j \\ z_{i}, z_{j} \in T_{2}}}\left|z_{i}-z_{j}\right| .
$$

To estimate the right hand side of (A.7), note that each factor of the form $d_{+}\left(z_{p}, e\right)^{1 / 2}$ where $z_{p} \in T_{1}$ and either $e \in T_{2}$ or $e=e^{\prime}$ is within a multiplicative constant of $\left|e^{\prime}\right|^{1 / 2}$. The number of such factors is $m_{1} m_{2}$. Also the same factors appear when $z_{p} \in T_{2}$ and $e \in T_{1}$ or $e=e^{\prime}$. Then the right hand side of A.7 is within a multiplicative constant of

$$
\left|e^{\prime}\right|^{m_{1} m_{2}} \prod_{e \in T_{1}} \prod_{z_{p} \in T_{1}} d_{+}\left(z_{p}, e\right)^{1 / 2} \prod_{e \in T_{2}} \prod_{z_{p} \in T_{2}} d_{+}\left(z_{p}, e\right)^{1 / 2}
$$

The estimate (A.7) now follows by induction.

Lemma A.6 Suppose $z_{1}, \ldots, z_{m}$ are in some bounded set $\mathcal{K}$. Let

$$
\Omega_{k}=\int_{e_{k}^{-}}^{e_{k}^{+}} \sqrt{\left(z-z_{1}\right) \ldots\left(z-z_{n}\right)} d z,
$$


so that $\Omega_{k}$ is the holonomy of the edge $e_{k}$. Then,

$$
\left|\frac{\partial\left(\Omega_{1}, \ldots, \Omega_{n-1}\right)}{\partial\left(\vec{e}_{1}, \ldots, \vec{e}_{m-1}\right)}\right| \leq C \prod_{i<j}\left|z_{i}-z_{j}\right|,
$$

where $C$ depends only on $m$ and $\mathcal{K}$.

Proof. Suppose for the moment that $z_{1}, \ldots, z_{m}$ are independent variables. We claim that

$$
\left|\frac{\partial \Omega_{k}}{\partial z_{j}}\right| \leq C \prod_{p=1}^{n} d_{+}\left(z_{p}, e_{k}\right)^{1 / 2},
$$

where $C$ depends only on $m$ and $\mathcal{K}$. Indeed,

$$
\frac{\partial \Omega_{k}}{\partial z_{j}}=\int_{e_{k}^{-}}^{e_{k}^{+}} \frac{\sqrt{\left(z-z_{1}\right) \ldots\left(z-z_{n}\right)}}{2\left(z-z_{j}\right)} d z
$$

If we write the numerator in the integral as $P\left(z-z_{j}\right)^{1 / 2}$, then $P$ is bounded by a constant times $\prod_{\substack{p=1 \\ p \neq j}}^{n} d_{+}\left(z_{p}, e_{k}\right)^{1 / 2}$. Then, the integral is bounded by

$$
\frac{|P|}{2}\left|\int_{e_{k}^{-}}^{e_{k}^{+}} \frac{d z}{\left(z-z_{j}\right)^{1 / 2}}\right| .
$$

If $d_{+}\left(z_{j}, e_{k}\right)>\frac{1}{2}\left|e_{k}^{+}-e_{k}^{-}\right|$,

$$
\left|\int_{e_{k}^{-}}^{e_{k}^{+}} \frac{d z}{\left(z-z_{j}\right)^{1 / 2}}\right| \leq C \frac{\left|e_{k}^{+}-e_{k}^{-}\right|}{d_{+}\left(z_{j}, e_{k}\right)^{1 / 2}} \leq C^{\prime}\left|e_{k}^{+}-e_{k}^{-}\right|^{1 / 2}
$$

(where we have used the assumption that $\left|e_{k}^{+}-e_{k}^{-}\right|$is bounded). If $d_{+}\left(z_{j}, e_{k}\right)<\frac{1}{2}\left|e_{k}^{+}-e_{k}^{-}\right|$, then

$$
\left|\int_{e_{k}^{-}}^{e_{k}^{+}} \frac{d z}{\left(z-z_{j}\right)^{1 / 2}}\right| \leq C^{\prime \prime}\left|e_{k}^{+}-e_{k}^{-}\right|^{1 / 2}
$$

where $C^{\prime \prime}$ depends only on $m$ and $\mathcal{K}$. Thus,

$$
\left|\frac{\partial \Omega_{k}}{\partial z_{j}}\right| \leq C\left|e_{k}^{+}-e_{k}^{-}\right|^{1 / 2} \prod_{p \neq j} d_{+}\left(z_{p}, e_{k}\right)^{1 / 2} .
$$

But by the choice of the tree $T$, for any $j, d_{+}\left(z_{j}, e_{k}\right) \geq c\left|e_{k}^{+}-e_{k}^{-}\right|$, where $c$ depends only on $m$. Thus, A.8 holds.

Since $\sum_{j=1}^{m} z_{j}=0$, there are $m-1$ linearly independent $z_{j}$ 's and therefore we can express $z_{j}=\sum_{k=1}^{m-1} \eta_{j k} \vec{e}_{k}$, where the $\eta_{j k}$ are bounded depending only on $m$. Then, we get

$$
\left|\frac{\partial \Omega_{j}}{\partial \vec{e}_{k}}\right| \leq C^{\prime} \prod_{p=1}^{m} d_{+}\left(z_{p}, e_{k}\right)^{1 / 2} .
$$


Lemma A.6 now follows from Lemma A.5.

Proof of Proposition A.4. It is enough to show that for any $\alpha,\left|\frac{\partial \mu}{\partial \beta_{\alpha}}\right| \leq C$ on $U_{\alpha}$. As above, we work with one zero of $q_{0}$ at a time. By (A.6) and Lemma A.6,

$$
\left|\frac{\partial\left(\Omega_{1}, \ldots, \Omega_{m-1}\right)}{\partial\left(a_{0}, \ldots, a_{m-2}\right)}\right|=\left|\frac{\partial\left(\Omega_{1}, \ldots, \Omega_{m-1}\right)}{\partial\left(\vec{e}_{1}, \ldots, \vec{e}_{m-1}\right)}\right|\left|\frac{\partial\left(\vec{e}_{1}, \ldots, \vec{e}_{m-1}\right)}{\partial\left(a_{0}, \ldots, a_{m-2}\right)}\right| \leq C
$$

where $C$ depends only on $m$ and $\mathcal{K}$.

Let $\mu^{\prime}=d \Omega_{1} d \bar{\Omega}_{1} \ldots d \Omega_{m-1} d \bar{\Omega}_{m-1}$, and let $\beta^{\prime}=d a_{0} d \bar{a}_{0} \ldots d a_{m-2} d \bar{a}_{m-2}$. Then, by (A.9),

$$
\frac{d \mu^{\prime}}{d \beta^{\prime}}=\left|\frac{\partial\left(\Omega_{1}, \ldots, \Omega_{m-1}\right)}{\partial\left(a_{0}, \ldots, a_{m-2}\right)}\right|^{2} \leq C^{2}
$$

Now,

$$
\frac{d \mu}{d \beta_{\alpha}}=\prod_{j} \frac{d \mu_{j}^{\prime}}{d \beta_{j}^{\prime}},
$$

where the product is over distinct zeroes of $q_{0}$. Now Proposition A.4 follows from A.9.

\section{References}

[Ac60] Robert D. M. Accola. Differentials and Extremal Length on Riemann Surfaces. Proceedings of the National Academy of Sciences of the United States of America, 46, No. 4. (Apr. 15, 1960), pp. 540-543.

[Bl61] C. Blatter. Über Extremallängen auf geschlossenen Flächen. (German) Comment. Math. Helv. 351961 153-168.

[CSF80] I. P. Cornfeld, Ya. G. Sinai, S. V. Fomin. Ergodic theory. "Nauka", Moscow, 1980.

[EM01] A. Eskin and H. Masur. Asymptotic formulas on flat surfaces. Ergodic Theory Dynam. Systems, 21 (2001), 443-478.

[EMc93] A. Eskin and C. McMullen. Mixing, counting, and equidistribution in Lie groups. Duke Math. J. 71 (1993), no. 1, 181-209.

[FaMa] B. Farb and D. Margalit. A primer on mapping class groups. http://www.math.utah.edu/ margalit/primer.

[FK] H. Farkas and I. Kra, Riemann surfaces. Graduate texts in Math.71, Springer-Verlag, New York, 1980.

[Fa73] J. D. Fay. Theta functions on Riemann surfaces. Lecture Notes in Mathematics 352, Springer 1973.

[FLP] A. Fathi, F. Laudenbach, and V. Poenaru, Travaux de Thurston sur les surfaces. Asterisque, Vols. 66 and 67 (1979). 
[Fo02] G. Forni. Deviation of ergodic averages for area-preserving flows on surfaces of higher genus. Ann. of Math. (2), 155 (2002), no. 1, 1-103.

[HP92] J. L. Harer and R. C. Penner. Combinatorics of Train Tracks. Annals of Math. Studies, 125, Princeton University Press, 1992.

[HuMas79] J. Hubbard, H. Masur. Quadratic differentials and foliations. Acta Math. 142 (1979), no. $3-4,221-274$.

[Ka90a] V. Kaimanovich. Bowen-Margulis and Patterson measures on negatively curved compact manifolds. Dynamical systems and related topics (Nagoya, 1990), 223-232, Adv. Ser. Dynam. Systems, 9, World Sci. Publ., River Edge, NJ, 1991.

[Ka90b] V. Kaimanovich. Invariant measures of the geodesic flow and measures at infinity on negatively curved manifolds. Hyperbolic behaviour of dynamical systems (Paris, 1990). Ann. Inst. H. Poincare Phys. Theor. 53 (1990), no. 4, 361-393.

[Ker80] S. Kerckhoff. The asymptotic geometry of Teichmüller space. Topology 19(1980), 23-41.

[Ko96] M. Kontsevich. Lyapunov exponents and Hodge theory. Mathematical Beauty of Physics, Saclay, 1996.

[KZ03] M. Kontsevich, A. Zorich. Connected components of the moduli spaces of Abelian differentials with prescribed singularities. Inventiones mathematicæ, 153 (2003), no.3, 631-678.

[LM] E. Lindenstrauss, M. Mirzakhani. Ergodic theory of the space of measured laminations. Int. Math. Res. Not. IMRN (2008), no. 4, Art. ID rnm126, 49 pp.

[Mar70] G. A. Margulis. On some aspects of the theory of Anosov flows. Ph.D. Thesis, 1970, Springer, 2003.

[Mas] B. Maskit, Comparison of hyperbolic and extremal lengths. Ann. Acad. Sci. Fenn., 10(1985), $381-386$.

[Mas76] H. Masur. Extension of the Weil-Peterson metric to the boundary of Teichmüller space. Duke Math. J. 43 (1976), no. 3, 623-635.

[Mas82] H. Masur. Interval exchange transformations and measured foliations. Ann. of Math. (2) 115 (1982), no. 1, 169-200.

[MasSm91] H. Masur and J. Smillie. Hausdorff Dimension of Sets of Nonergodic Measured Foliations Ann. of Math. (2) 134, No. 3 (1991), pp. 455-543

[Mi93] Y. Minsky. Teichmüller geodesics and ends of hyperbolic 3-manifolds. Topology, 32 (1993), 625-647.

[Mi96] Y. Minsky. Extremal length estimates and product regions in Teichmüller space. Duke Math. J. 83 (1996), no. 2, 249-286.

[Ra05] K. Rafi. Thick-thin decomposition of quadratic differentials. Math. Res. Lett. 14 (2007), no. $2,333-341$. 
[Ra07] K. Rafi. A combinatorial model for the Teichmüller metric. Geom. Funct. Anal., 17 (3), 936-959, 2007.

[Si94] Ya. G. Sinai. Topics in ergodic theory. Princeton University Press, 1994.

[Ve78] W. Veech. Interval exchange transformations. J. Analyse Math. 33 (1978), 222-272.

[Ve82] W. Veech. Gauss measures for transformations on the space of interval exchange maps. Ann. of Math. (2) 15(1982), 201-242.

[Ve86] W. Veech. The Teichmüller geodesic flow. Ann. of Math. (2) 124 (1986), no. 3, 441-530.

[Wo03] S. Wolpert. Geometry of the Weil-Petersson completion of Teichmüller space. Surveys in Differential Geometry, VIII: Papers in Honor of Calabi, Lawson, Siu and Uhlenbeck, editor S. T. Yau. International Press, Nov. 2003.

Department of Mathematics, University of Illinois, Urbana, IL 61801 USA;

jathreya@illinois.edu

Department of Mathematics, Rice University, MS 136, 6100 Main Street, Houston, Texas 772511892 and The Steklov Institute of Mathematics, Russian Academy of Sciences, Gubkina str. 8, 119991, Moscow, Russia;

aib1@rice.edu and bufetov@mi.ras.ru

Department of Mathematics, University of Chicago, Chicago IL 60637, USA;

eskin@math.uchicago.edu

Department of Mathematics, Stanford University, Stanford CA 94305 USA;

mmirzakh@math.stanford.edu 